\title{
Semi-Synthesis and Cytotoxicity Evaluation of Pyrimidine, Thiazole, and Indole Analogues of Argentatins A-C from Guayule (Parthenium Argentatum) Resin
}

Chandrashekhar Madasu

The University of Arizona

\section{Ya-Ming Xu}

The University of Arizona

E. M. Kithsiri Wijeratne

The University of Arizona

Manping X. Liu

The University of Arizona

István Molnár

The University of Arizona

Leslie GUNATILAKA ( $\square$ leslieg1@email.arizona.edu )

The University of Arizona https://orcid.org/0000-0001-9663-3600

\section{Research Article}

Keywords: guayule resin, argentatins A-C, pyrimidine analogues, thiazole analogues, indole analogues, cytotoxic activity

Posted Date: October 21st, 2021

DOl: https://doi.org/10.21203/rs.3.rs-975607/v1

License: (c) (i) This work is licensed under a Creative Commons Attribution 4.0 International License. Read Full License

Version of Record: A version of this preprint was published at Medicinal Chemistry Research on January 17th, 2022. See the published version at https://doi.org/10.1007/s00044-021-02835-1. 
Semi-synthesis and cytotoxicity evaluation of pyrimidine, thiazole, and indole analogues of argentatins A-C from guayule (Parthenium argentatum) resin

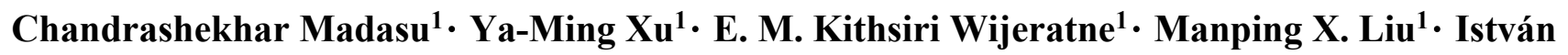

Molnár ${ }^{1}$ A. A. Leslie Gunatilaka ${ }^{1}$

$\square \quad$ A. A. Leslie Gunatilaka

leslieg1@email.arizona.edu

${ }^{1}$ Southwest Center for Natural Products Research, School of Natural Resources and the Environment, College of Agriculture and Life Sciences, University of Arizona, 250 E. Valencia Road, Tucson, Arizona 85706, USA 


\section{Abstract}

Argentatins A-C (1-3), the major cycloartane-type triterpenoids of guayule resin, a byproduct of commercial rubber production, were converted into their pyrimidine (7-12), thiazole (13-15), and indole (16-18) analogues by a molecular hybridization approach. The cytotoxic activities of these fused heterocyclic analogues 7-18 were compared with those of argentatins A-C (1-3) against a panel of three sentinel human cancer cell lines [NCI-H460 (non-small cell lung), MCF-7 (breast adenocarcinoma), and SF-268 (central nervous system glioma)], and normal human fibroblast (WI-38) cells. The cytotoxicity data suggest that the pyrimidine analogues $\mathbf{7}$ and $\mathbf{8}$ (derived from 1), $\mathbf{9}$ and $\mathbf{1 0}$ (derived from $\mathbf{2}$ ), and 12 (derived from 3) had significantly enhanced activity compared to the parent compounds or their thiazole (13-15) and indole (16-18) analogues. These findings indicate that triterpenoid constituents of guayule resin may be exploited to obtain value-added products with potential applications in anticancer drug discovery.

\section{Graphical abstract}

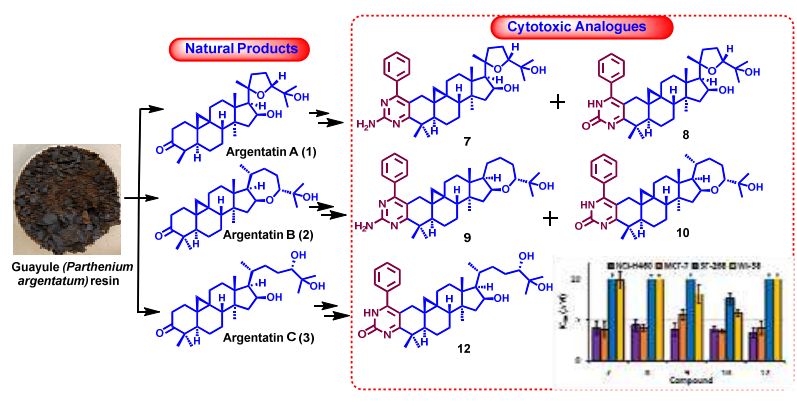

Keywords guayule resin; argentatins A-C; pyrimidine analogues; thiazole analogues; indole analogues; cytotoxic activity 


\section{Introduction}

Natural products (NPs) continue to be valuable sources of structurally diverse and biologically active compounds for drug discovery [1-3]. Many NPs have also served as promising lead compounds for drug development, especially as anticancer agents, after chemical modifications $[4,5]$. Triterpenoids, one of the major classes of NPs, have been reported to exhibit a variety of biological activities [6, 7]. Among these, cycloartane-type triterpenoids are found widely in plants and algae, some of which are known to display cytotoxic activity [8,9]. Cycloartane-type triterpenoids, such as argentatins A-C, have been found to be the major chemical components in Parthenium argentatum Gray (guayule) resin [10, 11], which is one of the byproducts in the manufacture of natural rubber from guayule, a widely-investigated arid land crop of potential industrial importance [12-14]. Among argentatins, argentatin A has been found to have antimicrobial activity [15], whereas argentatin B is known to inhibit proliferation of colon (HCT-15) and prostate (PC-3) cancer cells [16]. Detailed studies on argentatin B has revealed that it is a non-competitive inhibitor of 3H-estradiol-binding receptors of hormone-dependent breast cancer [17]. In addition, ring A modification of argentatins A and B have provided several analogues with enhanced cytotoxic activity against some selected cancer cell lines [18, 19]. Therefore, it was of interest to investigate the effect of structural modifications of argentatins A-C (1-3) on their potential anticancer activity for the purpose of obtaining value-added products from this byproduct of guayule rubber production.

Heteroaromatic moieties such as pyrimidines, thiazoles, and indoles are important fragments representing useful pharmacophores for the enhancement of therapeutic activity. Compounds containing these moieties have been found to exhibit a wide variety of biological activities, including anticancer, anti-inflammatory, antimicrobial, anti-HIV, anti-tubercular, antioxidant, and anti-hypertensive activities [20-25]. Molecular hybridization involving the linking of two structural moieties as a single hybrid is an important strategy in medicinal chemistry to enhance biological activity and pharmacokinetic properties 
$[26,27]$. We envisaged that argentatins A-C (1-3) with a carbonyl group at C-3 in ring A and some differences in ring D and the side chain could serve as suitable starting materials for conversion into the corresponding heteroaromatic hybrids which may then display enhanced biological activities. Herein we report the semi-synthesis of novel pyrimidine, thiazole, and indole analogues $\mathbf{7 - 1 8}$ of argentatins $\mathrm{A}-\mathrm{C}$ (1-3) and the investigation of their cytotoxic activity against three sentinel human cancer cell lines and normal human fibroblast cells.

\section{Results and discussion}

\section{Chemistry}

Argentatins A-C (1-3) obtained from guayule resin [28], were used for the synthesis of their target pyrimidine, thiazole, and indole analogues as outlined in Schemes 1 and 2. Depicted in Scheme 1 is the synthesis of pyrimidine analogues (7-12). Claisen-Schmidt condensation [22] of 1-3 with benzaldehyde afforded their corresponding benzylidene intermediates 4-6 in 74-77\% yield, which were the key precursors for the synthesis of their pyrimidine analogues. Presence of the benzylidene moiety in 4-6 were inferred from their characteristic absorption bands of the $\alpha, \beta$-unsaturated carbonyl group at 1678 $\mathrm{cm}^{-1}$ in their IR spectra and the presence of a signal at $\delta \mathrm{C} \sim 208$ in their ${ }^{13} \mathrm{C}$ NMR spectra. Intermediates 4-6 were separately treated with guanidine hydrochloride, thiourea, and urea in the presence of ethanolic $\mathrm{KOH}$ under reflux to give crude products which on treatment with DDQ (2,3-dichloro-5,6-dicyano- $p$ benzoquinone) in dry 1,4-dioxane at room temperature afforded 2'-amino-pyrimidine analogues (7, 9 , and 11) in 49-79\% yield and the 2'-oxo-3'H-pyrimidine analogues $(\mathbf{8}, \mathbf{1 0}$, and $\mathbf{1 2})$ in $31-58 \%$ yield. The structures of pyrimidine analogues were confirmed by the characteristic peaks in their ${ }^{13} \mathrm{C}$ NMR spectra. Signals around $\delta_{\mathrm{C}} 175,166,161$, and 116 ppm in 2'-amino-pyrimidine analogues 7, 9, and 11 were assigned to C-3, C-4', C-2' and C-2, respectively by the HMBC correlations of $\mathrm{H}_{3}-29 / \mathrm{C}-3, \mathrm{H}_{3}-30 / \mathrm{C}-3$, $\mathrm{H}_{2}-1 / \mathrm{C}-3, \mathrm{H}_{2}-1 / \mathrm{C}-4$ ', and $\mathrm{H}_{2}-1 / \mathrm{C}-2$. The presence of the 2'-amino-pyrimidine moiety in these was further 
confirmed by the ${ }^{1} \mathrm{H}$ NMR signal $\left(\delta_{\mathrm{H}} 4.80-4.90\right.$, brs, $\left.2 \mathrm{H}\right)$ due to the $\mathrm{NH}_{2}$ group. It is noteworthy that the pyrimidine analogues $\mathbf{8}, \mathbf{1 0}$, and 12 exhibited only two ${ }^{13} \mathrm{C}$ NMR signals for the pyrimidine moiety (at $\delta \mathrm{C} \sim 159$ and 110-112 ppm), assigned for C-2' and C-2 respectively, and the chemical shift of C-2 indicated the presence of 2'-oxo-substituted pyrimidine moiety in these [29]. The actual tautomer of pyrimidine moiety was suggested to be 3'H-pyrimidine because C-3 of $\mathbf{1 0}$ was detected at $\delta \mathrm{c} 164.4 \mathrm{ppm}$ by the HMBC experiment although the signals due to C-3 and C-4' of the pyrimidine ring were not detected or appeared as very weak peaks [30].

Synthesis of thiazole and indole analogues $(\mathbf{1 3}-\mathbf{1 8})$ of argentatins A-C (1-3) is outlined in Scheme 2. The target thiazole analogues were obtained by a two-step protocol. Compounds 1-3 were brominated at $\mathrm{C}-2$ by the reaction with liquid bromine in acetic acid at $0{ }^{\circ} \mathrm{C}-25{ }^{\circ} \mathrm{C}[18]$. The resulting mixture of bromoketones ( $\alpha$ and $\beta$ epimers) were used without further purification for the next step involving cyclization with thiourea [31] in ethanol at reflux temperature to afford fused amino-thiazoles (13-15) in 46-58\% yield. The identities of these products were confirmed by their NMR data. The characteristic broad singlet proton signals at $\delta_{\mathrm{H}} \sim 4.78 \mathrm{ppm}$ of these in their ${ }^{1} \mathrm{H}$ NMR spectra were assigned to the $\mathrm{NH}_{2}$ group, whereas the carbon signals in their ${ }^{13} \mathrm{C}$ NMR spectra at $\delta \mathrm{C} \sim 164-168\left(\mathrm{C}-2^{\prime}\right)$, $\sim 154$ (C-3), and $\sim 116$ (C-2) corresponding to thiazole rings were assigned with the help of HMBC correlations of $\mathrm{H}_{2}-1 / \mathrm{C}-3, \mathrm{H}_{2}-1 / \mathrm{C}-2$ and $\mathrm{H}_{3}-29 / \mathrm{C}-3, \mathrm{H}_{3}-30 / \mathrm{C}-3$.

The desired fused indole analogues (16-18) were prepared by Fries indole synthesis [31] involving condensation of argentatins A-C (1-3) with phenylhydrazine hydrochloride in acetic acid at $50{ }^{\circ} \mathrm{C}$. The structures of the resulting indole analogues were confirmed by their NMR data. In their ${ }^{1} \mathrm{H}$ NMR spectra, indole aromatic protons appeared as two pairs of doublets (H-2' 7.29 ppm; H-5' 7.38 ppm) and two pairs of triplets (H-3' 7.10 ppm; H-4' 7.04 ppm), and the NH protons appeared as singlets at $\delta_{\mathrm{H}} \sim 7.76 \mathrm{ppm}$. The ${ }^{13} \mathrm{C}$ NMR spectra exhibited eight characteristic signals in the range of $\delta \mathrm{C}$ 
108-143 ppm which were assigned by HMBC correlations of $\mathrm{H}_{2}-1 / \mathrm{C}-3, \mathrm{C}-2$ and C-7'; H-1'/C-2 and C7'; H-2'/C-4' and C-7'; H-3'/C-5' and C-6'; H-4'/C-2' and C-7'; H-5'/C-3' and C-6'; H3-29/C-3; and H330/C-3. The presence of cyclopropane ring of the argentatin moieties of 16-18 was confirmed by the proton signals at $\delta_{\mathrm{H}} \sim 0.5$ and 0.7 in their ${ }^{1} \mathrm{H}$ NMR spectra and the carbon signals at $\delta \mathrm{C} \sim 30$ in their ${ }^{13} \mathrm{C}$

NMR spectra. The structures of all synthesized compounds were further supported by their HRMS data.

\section{Cytotoxicity evaluation}

All twelve synthetic analogues (7-18) and their parent argentatins A-C (1-3) were evaluated for their cell proliferation inhibitory activity against a panel of three human sentinel cancer cell lines [NCI-H460 (non-small cell lung cancer), MCF-7 (breast adenocarcinoma), SF-268 (CNS cancer, glioma)] and normal human primary fibroblast (WI-38) cells by using the MTT assay [32]. Doxorubicin and DMSO were used as positive and negative controls, respectively. The results obtained are summarized in Table 1. These results revealed that compounds $7, \mathbf{8}, \mathbf{9}, \mathbf{1 0}$, and $\mathbf{1 2}$ were active below a $10 \mu \mathrm{M}$ concentration against most of the tested cell lines. Interestingly, amino-pyrimidine analogues 7 and 9 and oxopyrimidine analogues $\mathbf{8}, \mathbf{1 0}$, and 12 showed moderate cytotoxic effect against NCI-H460 and MCF-7 and were about 4-9-fold more active than the parent compounds 1-3. Additionally, the analogue 10 derived from argentatin B (2) showed moderate cytotoxicity against SF-268 cells and was ca. 4-fold more active than the parent compound. Significantly, most of the active compounds exhibited less cytotoxic activity towards the normal human fibroblast (WI-38) cells.

\section{Conclusion}

In summary, we designed and synthesized twelve new fused heterocyclic analogues 7-18 of argentatins A-C (1-3) by utilizing the C-3 keto functionality of ring A of the parent compounds. All analogues were evaluated for their cell proliferation inhibitory activity against the three sentinel cancer cell lines NCIH460, MCF-7, SF-268, and towards normal fibroblast cells, WI-38. The bioassay results demonstrated 
that the analogues 7, 8, 9, 10, and 12 exhibited enhanced cytotoxic activity against NCI-H460 and MCF7 cells compared to the parent argentatins $\mathbf{1}-\mathbf{3}$. Among all compounds tested, the pyrimidine analogue 10 was unique in displaying single-digit micro-molar activity against SF-268 cells. The preliminary structure-activity relationship (SAR) study suggested that the 2'-amino-pyrimidine and 2'-oxo-3'Hpyrimidine derivatives were more active than the thiazole and the indole analogues against the cell lines tested. These findings support the possibility of development of potential anticancer agents from some triterpenoid constituents of the guayule resin.

\section{Experimental}

\section{Chemistry}

\section{General}

Reagents for chemical synthesis were purchased from Sigma-Aldrich and Fischer Scientific (USA). All solvents were distilled prior to use. The progress of all reactions was monitored by thin-layer chromatography (TLC) using silica gel $60 \mathrm{~F}_{254}$ plates (Merck). Visualization was accomplished under UV light (254 nm) and spraying with $p$-anisaldehyde in $\mathrm{H}_{2} \mathrm{SO}_{4} / \mathrm{H}_{2} \mathrm{O} / \mathrm{CH}_{3} \mathrm{CO}_{2} \mathrm{H}$ followed by heating. Purification of compounds was carried out by column chromatography using silica gel $40 \mu \mathrm{m}$ flash chromatography packing (J. T. Baker, Jackson, TN, USA). 1D and 2D NMR spectra were recorded in $\mathrm{CDCl}_{3}$ with a Bruker Avance III 400 spectrometer at $400 \mathrm{MHz}$ for ${ }^{1} \mathrm{H}$ NMR and $100 \mathrm{MHz}$ for ${ }^{13} \mathrm{C}$ NMR using residual $\mathrm{CHCl}_{3}$ as the internal standard. The chemical shift $(\delta)$ values are expressed in parts per million (ppm) and coupling constants $(J)$ are given in Hertz $(\mathrm{Hz})$. Optical rotations were measured with a JASCO Dip-370 polarimeter using $\mathrm{MeOH}$ as the solvent. Preparative HPLC purification was performed on a Waters Delta Prep 4000 equipped with a PDA 996 detector using a $10 \times 250 \mathrm{~mm}$ Phenomenex Luna $5 \mu \mathrm{m}$ C-18 column for reversed-phase (RP) chromatography. High Resolution Mass Spectra (HRMS) were recorded on Agilent G6224A TOF mass spectrometer. The following 
abbreviations were used to indicate the NMR signals: $\mathrm{s}=$ singlet, brs $=$ broad singlet, $\mathrm{d}=$ doublet, $\mathrm{t}=$ triplet, $\mathrm{m}=$ multiplet, $J=$ coupling constant.

\section{Isolation and identification of argentatins A-C (1-3)}

Argentatins A-C (1-3) used for the semi-synthesis of their analogues 7-18 were obtained from guayule (Parthenium argentatum AZ-2) resin as described previously [26].

\section{General procedure for synthesis of compounds 4-6}

To a solution of appropriate compounds 1 (1.0 eq, $50.0 \mathrm{mg}), 2(1.0 \mathrm{eq}, 50.0 \mathrm{mg})$, and $3(1.0 \mathrm{eq}, 60.0 \mathrm{mg})$ in absolute $\mathrm{EtOH}(5.0 \mathrm{~mL})$ were added benzaldehyde (2.0 eq) and $\mathrm{KOH}(2.0 \mathrm{eq})$, and the resulting mixture was stirred at $45^{\circ} \mathrm{C}$ for $12 \mathrm{~h}$. After completion of the reaction (monitored by TLC), it was neutralized with 10\% aq. $\mathrm{HCl}$ and extracted $(3 \mathrm{x})$ with EtOAc. The combined organic layer was dried over anhydrous $\mathrm{Na}_{2} \mathrm{SO}_{4}$, evaporated under reduced pressure, and the residue was passed through a silica gel column with $\mathrm{CH}_{2} \mathrm{Cl}_{2}-i$-PrOH (97:3) and hexane-EtOAc (85:15) as eluents to afford 4 (44.0 mg, 74\%), 5 (46.0 mg, 77\%), and 6 (46.6 mg, 79\%) respectively.

2-Benzylidene-(16 $\beta, 20 S, 24 R)$-20,24-epoxy-16,25-dihydroxycycloartan-3-one (4) Light yellow solid; $[\alpha]_{\mathrm{D}}^{25}+136.0^{\circ}\left(c\right.$ 0.1, $\left.\mathrm{CHCl}_{3}\right) ; \mathrm{IR}(\mathrm{KBr}) v_{\max } 3394(\mathrm{O}-\mathrm{H}), 2970(\mathrm{C}-\mathrm{H}), 1678$ ( $\alpha, \beta$-unsaturated ketone), $1593(\mathrm{Ar} C=\mathrm{C}), 1461,1380,1164(\mathrm{C}-\mathrm{O}), 1018,952,694 \mathrm{~cm}^{-1} ;{ }^{1} \mathrm{H}$ NMR $\left(400 \mathrm{MHz}, \mathrm{CDCl}_{3}\right): \delta 0.44(\mathrm{~d}$, $J=4.6 \mathrm{~Hz}, 1 \mathrm{H}, \mathrm{H}-19 \mathrm{a}), 0.70$ (d, $J=4.6 \mathrm{~Hz}, 1 \mathrm{H}, \mathrm{H}-19 \mathrm{~b}), 0.91$ (s, 3H, H3-28), 0.92 (m, 1H, H-6a), 1.07 (s, 3H, H3-29), 1.21 (m, 1H, H-7a), 1.11 (s, 3H, H3-26), 1.17 (s, 3H, H3-30), 1.22 (s, 3H, H3-27), 1.25 (s, 3H, $\left.\mathrm{H}_{3}-18\right), 1.38$ (m, 1H, H-7b), 1.40 (s, 3H, H3-21), 1.51 (m, 1H, H-15a), 1.60 (m, 1H, H-8), 1.64 (m, 1H, H-6b), 1.70 (m, 1H, H-22a), 1.76 (m, 2H, H2-12), 1.98 (m, 1H, H-5), 1.94 (m, 2H, H2-23), 2.03 (m,

1H, H-15b), 2.13 (d, $J=7.8 \mathrm{~Hz}, 1 \mathrm{H}, \mathrm{H}-17), 2.16$ (m, 2H, H2-11), 2.24 (m, 1H, H-22b), 2.51 (d, $J=16.2$ Hz, 1H, H-1a), 2.85 (d, J=16.2 Hz, 1H, H-1b), 3.82 (t, J=15.0 Hz, 1H, H-24), 4.59 (m, 1H, H-16), 7.27-7.36 (m, 5H, Ar-H), 7.37 (s, 1H, H-2'); ${ }^{13} \mathrm{C}$ NMR (100 MHz, CDCl $): \delta 208.1(\mathrm{C}-3), 135.9\left(\mathrm{C}-2^{\prime}\right)$, 
135.8 (C-2), 130.1 (Ar-C), 128.3 (Ar-C), 128.2 (Ar-C), 87.1 (C-20), 84.5 (C-24), 73.3 (C-16), 70.8 (C25), 55.6 (C-17), 48.7 (C-4), 48.6 (C-15), 48.4 (C-8), 46.5 (C-14), 46.3 (C-13), 45.4 (C-5), 37.3 (C-22), 36.6 (C-1), 33.1 (C-12), 30.3 (C-19), 27.3 (C-27), 26.2 (C-11), 26.1 (C-26), 25.5 (C-7), 25.3 (C- 18), 24.2 (C-30), 24.1 (C-10), 23.7 (C-23), 21.9 (C-6), 21.3 (C-29), 21.1 (C-21), 20.5 (C-28), 19.3 (C-9); HRESIMS $m / z$ calcd for $\mathrm{C}_{37} \mathrm{H}_{53} \mathrm{O}_{4}$ 561.3941, found $561.3938[\mathrm{M}+\mathrm{H}]^{+}$.

2-benzylidene-(16 $\beta, 20 R, 24 R)$-16,24-epoxy-25-hydroxy-cycloartan-3-one (5) Light yellow solid; $[\alpha]_{\mathrm{D}}^{25}$ $+109.5^{\circ}\left(c\right.$ 0.1, $\left.\mathrm{CHCl}_{3}\right)$; IR (KBr) v $\max 3409$ (O-H), 2939 (C-H), 1678 ( $\alpha, \beta$ unsaturated ketone), 1593 $(\mathrm{Ar} \mathrm{C}=\mathrm{C}), 1461,1377,1164(\mathrm{C}-\mathrm{O}), 1033,694 \mathrm{~cm}^{-1} ;{ }^{1} \mathrm{H} \mathrm{NMR}\left(400 \mathrm{MHz}, \mathrm{CDCl}_{3}\right): \delta 0.43(\mathrm{~d}, J=4.0 \mathrm{~Hz}$, 1H, H-19a), 0.70 (d, J=4.0 Hz, 1H, H-19b), 0.90 (s, 3H, H3-28), 0.91 (d, J=6.4 Hz, 3H, H3-21), 1.03 (m, 1H, H-7a), 1.08 (s, 3H, H3-29), 1.07 (s, 6H, H3-26, H3-27), 1.13 (s, 3H, H3-18), 1.17 (s, 3H, $\left.\mathrm{H}_{3}-30\right)$, 1.38 (m, 1H, H-7b), 1.39 (m, 1H, H-22a), 1.50 (m, 1H, H-15a), 1.52 (m, 1H, H-23a), 1.60 (m, 1H, H-8), 1.62 (m, 1H, H-17), 1.63 (m, 2H, H2-6), 1.64 (m, 2H, H2-12), 1.74 (m, 1H, H-22b), 1.87 (m, 1H, H15b), 1.92 (m, 1H, H-23b), 1.98 (m, 1H, H-5), 2.05 (m, 1H, H-20), 2.14 (m, 2H, H2-11), 2.52 (d, $J=$ 16.6 Hz, 1H, H-1a), 2.85 (d, $J=16.6$ Hz, 1H, H-1b), 3.57 (dd, 1H, H-24), 4.60 (m, 1H, H-16), 7.27-7.36 (m, 5H, Ar-H), 7.37 (s, 1H, H-2'); ${ }^{13} \mathrm{C}$ NMR (100 MHz, $\left.\mathrm{CDCl}_{3}\right): \delta 208.2(\mathrm{C}-3), 135.9$ (Ar-C, C-2'), 135.8 (C-2), 130.1 (Ar-C), 128.3 (Ar-C), 128.2 (Ar-C), 82.5 (C-24), 74.8 (C-16), 73.2 (C-25), 57.4 (C17), 48.6 (C-4), 48.1 (C-8), 45.8 (C-14), 45.6 (C-13), 45.3 (C-5), 45.0 (C-15), 36.7 (C-1), 35.4 (C-22), 32.7 (C-12), 30.0 (C-19), 29.0 (C-20), 25.6 (C-11), 25.9 (C-27), 25.6 (C-7), 24.2 (C-30), 24.3 (C-10), 23.8 (C-26), 23.4 (C-23), 21.9 (C-6), 21.3 (C-29), 20.9 (C-21), 19.7 (C-28), 19.4 (C-9), 19.0 (C- 18); HRESIMS $m / z$ calcd for $\mathrm{C}_{37} \mathrm{H}_{53} \mathrm{O}_{3}$ 545.3938, found $545.3989[\mathrm{M}+\mathrm{H}]^{+}$.

2-benzylidene-(16 $\beta, 20 R, 24 R)$-16,24,25-trihydroxy-cycloartan-3-one (6) Light yellow solid; $[\alpha]_{\mathrm{D}}^{25}$ $+148.0^{\circ}$ ( c 0.1, MeOH); IR (KBr) v $\max 3421(\mathrm{O}-\mathrm{H}), 2943(\mathrm{C}-\mathrm{H}), 1678$ ( $\alpha$, $\beta$-unsaturated ketone), 1596 $(\mathrm{Ar} C=\mathrm{C}), 1458,1377,1157(\mathrm{C}-\mathrm{O}), 1026,694 \mathrm{~cm}^{-1} ;{ }^{1} \mathrm{H} \mathrm{NMR}\left(400 \mathrm{MHz}, \mathrm{CDCl}_{3}\right): \delta 0.44(\mathrm{~d}, J=4.2 \mathrm{~Hz}$, 1H, H-19a), 0.70 (d, J=4.2 Hz, 1H, H-19b), 0.90 (d, J=6.4 Hz, 3H, H3-21), 0.91 (s, 3H, H3-28), 1.07 
(s, 3H, H3-29), 1.09 (m, 1H, H-22a), 1.13 (s, 6H, H3-18, H3-26, ), 1.17 (s, 3H, H3-30), 1.20 (s, 3H, H327), 1.33 (m, 1H, H-15a), 1.37 (m, 2H, H2-7), 1.40 (m, 1H, $\left.\mathrm{H}_{2}-23\right), 1.57$ (m, 1H, H-8), 1.62 (m, 2H, $\mathrm{H}_{2}-$ 6), 1.64 (m, 1H, H-17), 1.66 (m, 2H, H2-12), 1.80 (m, 1H, H-22b), 1.90 (m, 1H, H-20), 1.98 (m, 1H, H5), 2.06 (m, 1H, H-15b), 2.13 (m, 2H, H2-11), 2.52 (d, J=16.4 Hz, 1H, H-1a), 2.85 (d, J=16.4 Hz, 1H, H-1b), 3.55 (dd, 1H, H-24), 4.48 (m, 1H, H-16), 7.27-7.36 (m, 5H, Ar-H), 7.37 (s, 1H, H-2'); ${ }^{13} \mathrm{C}$ NMR (100 MHz, $\mathrm{CDCl}_{3}$ ): $\delta 208.2$ (C-3), 135.9 (C-2'), 135.8 (C-2), 130.1 (Ar-C), 128.3 (Ar-C), 128.2 (Ar-C), 75.2 (C-24), 73.0 (C-16), 72.8 (C-25), 56.8 (C-17), 48.6 (C-8), 48.5 (C-4), 47.9 (C-15), 46.6 (C-14), 45.3 (C-5), 45.2 (C-13), 36.6 (C-1), 32.6 (C-12), 31.1 (C-22), 30.1 (C-19), 26.8 (C-27), 26.7 (C-20), 26.5 (C-11), 26.0 (C-7), 25.6 (C-23), 25.3 (C-10), 24.3 (C-30), 22.9 (C-26), 22.0 (C-6), 21.9 (C-29), 20.1 (C-28), 19.5 (C-9), 19.2 (C-18), 17.6 (C-21). HRESIMS $m / z$ calcd for $\mathrm{C}_{37} \mathrm{H}_{55} \mathrm{O}_{4} 563.4078$, found $563.4094[\mathrm{M}+\mathrm{H}]^{+}$.

\section{Synthesis of argentatin analogues 7, 9 and 11}

To stirred solutions of appropriate intermediates $4(1.0 \mathrm{eq}, 15.0 \mathrm{mg}), \mathbf{5}(1.0 \mathrm{eq}, 10.0 \mathrm{mg})$ and $\mathbf{6}(1.0 \mathrm{eq}$, $20.0 \mathrm{mg})$ in absolute EtOH $(1.5 \mathrm{~mL})$ were added guanidine hydrochloride (2.0 eq) and $\mathrm{KOH}(2.0 \mathrm{eq})$, and the resulting reaction mixture was refluxed for $12 \mathrm{~h}$ (monitored by TLC). The reaction mixtures were neutralized with $10 \%$ aq. $\mathrm{HCl}$ and extracted with EtOAc $(3 \times 5 \mathrm{~mL})$. The combined organic layers were dried over anhydrous $\mathrm{Na}_{2} \mathrm{SO}_{4}$ and concentrated under reduced pressure to give crude products. Crude products were then dissolved in dry 1,4-dioxane (2.0 mL), DDQ (1.5 eq) was added and the resulting reaction mixtures were stirred at $25^{\circ} \mathrm{C}$ for $1 \mathrm{~h}$ (TLC control). The reaction mixtures were then quenched with $10 \%$ aq. $\mathrm{NaHCO}_{3}$ solution, and extracted with EtOAc $(3 \times 5 \mathrm{~mL})$ and evaporated under vacuo. The resulting residues were purified by column chromatography using silica gel with tolueneEtOAc (90:10) and $\mathrm{CH}_{2} \mathrm{Cl}_{2}-i$ - $\mathrm{PrOH}(95: 5)$ to afford the argentatin analogues 7 (12.8 mg, $\left.79 \%\right), 9$ (6.4 $\mathrm{mg}, 60 \%)$, and $11(10.5 \mathrm{mg}, 49 \%)$. 
$(16 \beta, 20 S, 24 R)$-20,24-epoxy-16,25-dihydroxy-2'-amino-4'-phenylpyrimidino[5',6':2,3]cycloartane (7) White solid; $[\alpha]_{\mathrm{D}}^{25}+162.4^{\circ}\left(c\right.$ 0.1, MeOH); IR (KBr) $v_{\max } 3495(\mathrm{O}-\mathrm{H}), 3402(\mathrm{~N}-\mathrm{H}), 2939(\mathrm{C}-\mathrm{H}), 1608,1550(\mathrm{Ar}$ $\mathrm{C}=\mathrm{C}), 1450,1369,1180(\mathrm{C}-\mathrm{O}), 1056 \mathrm{~cm}^{-1} ;{ }^{1} \mathrm{H}$ NMR $\left(400 \mathrm{MHz}, \mathrm{CDCl}_{3}\right): \delta 0.32(\mathrm{~d}, J=4.3 \mathrm{~Hz}, 1 \mathrm{H}, \mathrm{H}-19 \mathrm{a})$, 0.64 (d, $J=4.3 \mathrm{~Hz}, 1 \mathrm{H}, \mathrm{H}-19 \mathrm{~b}), 0.89$ (s, 3H, H3-28), 0.96 (m, 1H, H-6a), 1.13 (m, 1H, H-7a), 1.10 (s, 3H, H3-26), 1.19 (s, 3H, $\left.\mathrm{H}_{3}-30\right), 1.21$ (s, 3H, $\left.\mathrm{H}_{3}-18\right), 1.22$ (s, 3H, $\left.\mathrm{H}_{3}-27\right), 1.38$ (m, 1H, H-7b), 1.37 (s, 3H, $\mathrm{H}_{3}-29$ ), 1.38 (s, 3H, H3-21), 1.49 (m, 1H, H-15a), 1.67 (m, 2H, H2-12), 1.66 (m, 1H, H-22a), 1.67 (m, 1H, H-8), 1.68 (m, 1H, H-5), 1.78 (m, 1H, H-6b), 1.92 (m, 2H, Hz-23), 1.99 (d, J=16.0 Hz, 1H, H-1a), 2.05 (m, 1H, H15b), 2.02 (m, 2H, H2-11), 2.12 (d, $J=7.6 \mathrm{~Hz}, 1 \mathrm{H}, \mathrm{H}-17), 2.23$ (m, 1H, H-22b), 2.85 (d, $J=16.0 \mathrm{~Hz}, 1 \mathrm{H}, \mathrm{H}-$ 1b), 3.82 (t, $J=15.2 \mathrm{~Hz}, 1 \mathrm{H}, \mathrm{H}-24), 4.57$ (q, 1H, H-16), 4.87 (s, 2H, - $\left.\mathrm{NH}_{2}\right), 7.35-7.43$ (m, 5H, Ar-H); ${ }^{13} \mathrm{C}$ NMR (100 MHz, CDCl $): \delta 175.3$ (C-3), $166.5(\mathrm{C}-4$ '), 161.0 (C-2'), 139.0 (Ar-C), 128.5 (Ar-C), 128.3 (ArC), 128.2 (Ar-C), 115.8 (C-2), 87.2 (C-20), 84.5 (C-24), 73.4 (C-16), 70.9 (C-25), 55.6 (C-17), 48.5 (C-5), 48.4 (C-15), 46.2 (C-8), 46.5 (C-14), 45.8 (C-13), 42.3 (C-4), 37.3 (C-22), 34.9 (C-1), 33.1 (C-12), 30.0 (C19), 27.4 (C-29), 27.3 (C-27), 26.1 (C-26), 25.8 (C-11), 25.6 (C-18), 25.3 (C-10), 25.2 (C-7), 24.4 (C-30), 23.7 (C-23), 22.3 (C-6), 21.2 (C-21), 20.6 (C-28), 18.9 (C-9); HRESIMS m/z calcd for $\mathrm{C}_{38} \mathrm{H}_{54} \mathrm{~N}_{3} \mathrm{O}_{3} 600.4120$, found $600.4156[\mathrm{M}+\mathrm{H}]^{+}$.

(16ß,20R,24R)-16,24-epoxy-25-hydroxy-2'-amino-4'-phenyl-pyrimidino[5',6':2,3]cycloartane (9) White solid; $[\alpha]_{\mathrm{D}}^{25}+31.5^{\circ}\left(c\right.$ 0.1, MeOH); IR (KBr) $v_{\max } 3490(\mathrm{O}-\mathrm{H}), 3375(\mathrm{~N}-\mathrm{H}), 2939(\mathrm{C}-\mathrm{H}), 1612,1550(\mathrm{Ar}$ $\mathrm{C}=\mathrm{C}), 1442,1369,1168(\mathrm{C}-\mathrm{O}), 1060,702 \mathrm{~cm}^{-1} ;{ }^{1} \mathrm{H}$ NMR $\left(400 \mathrm{MHz}, \mathrm{CDCl}_{3}\right) \delta 0.32(\mathrm{~d}, J=4.4 \mathrm{~Hz}, 1 \mathrm{H}$, H-19a), 0.63 (d, $J=4.4 \mathrm{~Hz}, 1 \mathrm{H}, \mathrm{H}-19 \mathrm{~b}), 0.87$ (d, $\left.J=6.4 \mathrm{~Hz}, 3 \mathrm{H}, \mathrm{H}_{3}-21\right), 0.89$ (s, 3H, H3-28), 1.07 (s, 6H, $\left.\mathrm{H}_{3}-26, \mathrm{H}_{3}-27\right), 1.10$ (s, 3H, H3-18), 1.16 (m, 1H, H-7a), 1.19 (s, 3H, H3-30), 1.38 (m, 1H, H-22a), 1.40 (m, 1H, H-7b), 1.48 (m, 1H, H-15a), 1.37 (s, 3H, H3-29), 1.54 (m, 1H, H-23a), 1.76 (m, 2H, H2-6), 1.57 (m, 1H, H-17), 1.60 (m, 2H, H2-12), 1.55 (m, 1H, H-5), 1.85 (m, 1H, H-8), 1.74 (m, 1H, H-22b), 1.97 (d, $J=16.2 \mathrm{~Hz}, 1 \mathrm{H}, \mathrm{H}-1 \mathrm{a}), 1.88$ (m, 1H, H-15b), 1.90 (m, 1H, H-23b), 2.02 (m, 2H, H2-11), 2.03 (m, 1H, H-20), 2.84 (d, J=16.2 Hz, 1H, H-1b), 3.56 (dd, 1H, H-24), 4.57 (m, 1H, H-16), 4.85 (Brs, 2H, 
-NH2), 7.33-7.45 (m, 5H, Ar-H); ${ }^{13} \mathrm{C}$ NMR (100 MHz, $\left.\mathrm{CDCl}_{3}\right) \delta 175.2(\mathrm{C}-3), 166.6(\mathrm{C}-4$ '), $160.9(\mathrm{C}-$ 2'), 138.9 (Ar-C), 128.5 (Ar-C), 128.3 (Ar-C), 128.2 (Ar-C), 115.9 (C-2), 82.5 (C-24), 74.8 (C-16), 73.2 (C-25), 57.4 (C-17), 48.5 (C-5), 45.8 (C-14), 45.6 (C-8), 45.6 (C-13), 45.1 (C-15), 42.3 (C-4), 35.4 (C22), 34.9 (C-1), 32.6 (C-12), 29.8 (C-19), 28.8 (C-20), 27.4 (C-29), 25.7 (C-7), 25.6 (C-10), 25.4 (C-27), 24.3 (C-11), 23.8 (C-26), 23.6 (C-23), 23.4 (C-30), 22.3 (C-6), 20.8 (C-21), 19.7 (C-9), 19.0 (C-28), 19.0 (C- 18); HRESIMS $m / z$ calcd for $\mathrm{C}_{38} \mathrm{H}_{54} \mathrm{~N}_{3} \mathrm{O}_{2} 584.4216$, found $584.4214[\mathrm{M}+\mathrm{H}]^{+}$.

$(16 \beta, 20 R, 24 R)-16,24,25-t r i h y d r o x y-2$ '-amino-4'-phenyl-pyrimidino[5',6':2,3]cycloartane (11) White solid; $[\alpha]_{\mathrm{D}}^{25}+127.0^{\circ}\left(c\right.$ 0.1, MeOH); IR (KBr) $v_{\max } 3504(\mathrm{O}-\mathrm{H}), 3406(\mathrm{~N}-\mathrm{H}), 2933(\mathrm{C}-\mathrm{H}), 1622,1556(\mathrm{Ar}$ $\mathrm{C}=\mathrm{C}), 1456,1380,1022,702 \mathrm{~cm}^{-1} ;{ }^{1} \mathrm{H}$ NMR (400 MHz, $\left.\mathrm{CDCl}_{3}\right) \delta 0.32(\mathrm{~d}, J=4.2 \mathrm{~Hz}, 1 \mathrm{H}, \mathrm{H}-19 \mathrm{a}), 0.64$ (d, $J=4.2 \mathrm{~Hz}, 1 \mathrm{H}, \mathrm{H}-19 \mathrm{~b}), 0.87$ (d, $\left.J=6.3 \mathrm{~Hz}, 3 \mathrm{H}, \mathrm{H}_{3}-21\right), 0.90$ (s, 3H, H3-28), 1.12 (s, 3H, H3-26), 1.14 (s, 3H, H3-18), 1.18 (m, 1H, H-22a), 1.19 (s, 6H, H3-30, H3-27), 1.20 (m, 1H, H-23a), 1.38 (m, $1 \mathrm{H}$, H-15a), 1.42 (m, 1H, H-23b), 1.37 (s, 3H, H3-29), 1.59 (m, 2H, H2-6), 1.60 (m, 1H, H-5), 1.62 (m, 2H, H2-12), 1.64 (m, 1H, H-17), 1.77 (m, 1H, H-22b), 1.80 (m, 2H, H2-7), 1.86 (m, 1H, H-20), 1.98 (d, $J=$ $16.5 \mathrm{~Hz}, 1 \mathrm{H}, \mathrm{H}-1 \mathrm{a}), 1.97$ (m, 1H, H-8), 2.04 (m, 2H, H2-11), 2.08 (m, 1H, H-15b), 2.85 (d, J=16.2 Hz, 1H, H-1b), 3.55 (dd, 1H, H-24), 4.47 (m, 1H, H-16), 4.82 (brs, 2H, -NH2), 7.33-7.43 (m, 5H, Ar-H); ${ }^{13} \mathrm{C}$ NMR (100 MHz, $\left.\mathrm{CDCl}_{3}\right) \delta 175.3$ (C-3), $166.5(\mathrm{C}-4$ '), 160.8 (C-2'), 138.6 (Ar-C), 128.6 (Ar-C), 128.1 (Ar-C), 115.8 (C-2), 75.2 (C-24), 72.8 (C-25), 72.6 (C-16), 56.7 (C-17), 48.8 (C-5), 47.5 (C-15), 46.4 (C-8), 45.6 (C-14), 45.1 (C-13), 42.3 (C-4), 34.8 (C-1), 32.5 (C-12), 31.1 (C-22), 29.6 (C-19), 27.2 (C27), 26.5 (C-20), 26.3 (C-11), 26.1 (C-7), 25.6 (C-23), 25.5 (C-10), 24.2 (C-26), 23.5 (C-29), 22.7 (C-6), 22.2 (C-30), 20.0 (C-9), 19.0 (C- 18, C-28), 17.6 (C-21); HRESIMS m/z calcd for $\mathrm{C}_{38} \mathrm{H}_{56} \mathrm{~N}_{3} \mathrm{O}_{3}$ 602.4322, found $602.4344[\mathrm{M}+\mathrm{H}]^{+}$. 


\section{Synthesis of argentatin analogues 8, 10 and 12}

To stirred solutions of the appropriate intermediates $4(1.0 \mathrm{eq}, 15.0 \mathrm{mg}), \mathbf{5}(1.0 \mathrm{eq}, 16.0 \mathrm{mg})$, and $\mathbf{6}(1.0$ eq, $15.0 \mathrm{mg})$ in absolute $\mathrm{EtOH}(1.5 \mathrm{~mL})$ were added urea $(2.0 \mathrm{eq})$ and $\mathrm{KOH}(2.0 \mathrm{eq})$, and the resulting mixtures were refluxed for $12 \mathrm{~h}$ (TLC control). The reaction mixtures were then neutralized with $10 \%$ aq. $\mathrm{HCl}$ and extracted with EtOAc $(3 \times 5 \mathrm{~mL})$. The combined organic layers were dried over anhydrous $\mathrm{Na}_{2} \mathrm{SO}_{4}$ and concentrated under reduced pressure. The resulting residues were dissolved in dry 1,4dioxane $(2.0 \mathrm{~mL})$, DDQ $(1.5 \mathrm{eq})$ was added and stirred at $25^{\circ} \mathrm{C}$ for $1 \mathrm{~h}$ (TLC control). The reaction mixtures were quenched with $10 \%$ aq. $\mathrm{NaHCO}_{3}$ solution, extracted with EtOAc $(3 \times 5 \mathrm{~mL})$, dried (anhydrous $\mathrm{Na}_{2} \mathrm{SO}_{4}$ ), and evaporated under reduced pressure to provide the crude products which were then purified by column chromatography using silica gel eluting with toluene-EtOAc (15:10) and $\mathrm{CH}_{2} \mathrm{Cl}_{2}-i-\mathrm{PrOH}(95: 10)$ to afford the argentatin analogues 8 (5.0 mg, 31\%), $\mathbf{1 0}(10.0 \mathrm{mg}, 58 \%)$, and $\mathbf{1 2}$ (6.5 mg, 40\%).

$(16 \beta, 20 S, 24 R)-20,24-e p o x y-16,25$-dihydroxy-2'-oxo-4'-phenyl-3'H-pyrimidino[5',6':2,3] cycloartane (8)

White solid; $[\alpha]_{\mathrm{D}}^{25}+20.7^{\circ}(c 0.05, \mathrm{MeOH})$; IR (KBr) $v_{\max } 3429(\mathrm{OH}), 2941(\mathrm{C}-\mathrm{H}), 1645(\mathrm{C}=\mathrm{O}), 1600(\mathrm{Ar}$ $\mathrm{C}=\mathrm{C}), 1450,1379,1110(\mathrm{C}-\mathrm{O}), 1056 \mathrm{~cm}^{-1} ;{ }^{1} \mathrm{H}$ NMR $\left(400 \mathrm{MHz}, \mathrm{CDCl}_{3}\right) \delta 0.34(\mathrm{~d}, J=4.3 \mathrm{~Hz}, 1 \mathrm{H}, \mathrm{H}-$ 19a), 0.65 (d, J=4.3 Hz, 1H, H-19b), 0.88 (s, 3H, H3-28), 0.96 (m, 1H, H-6a), 1.13 (m, 1H, H-7a), 1.10 (s, 3H, H3-26), 1.21 (s, 6H, H3-18, H3-30), 1.39 (m, 1H, H-7b), 1.37 (s, 6H, H3-27, H3-29), 1.51 (s, 3H, H3-21), 1.49 (m, 1H, H-15a), 1.67 (m, 2H, H2-12), 1.66 (m, 1H, H-22a), 1.67 (m, 1H, H-8), 1.68 (m, 1H, H-5), 1.78 (m, 1H, H-6b), 1.92 (m, 2H, H2-23), 1.87 (d, $J=16.0$ Hz, 1H, H-1a), 2.05 (m, 1H, H-15b), $2.02\left(\mathrm{~m}, 2 \mathrm{H}, \mathrm{H}_{2}-11\right), 2.10$ (d, $\left.J=7.6 \mathrm{~Hz}, 1 \mathrm{H}, \mathrm{H}-17\right), 2.22(\mathrm{~m}, 1 \mathrm{H}, \mathrm{H}-22 \mathrm{~b}), 2.80$ (d, $J=16.0 \mathrm{~Hz}, 1 \mathrm{H}, \mathrm{H}-$ 1b), 3.81 (t, $J=15.2 \mathrm{~Hz}, 1 \mathrm{H}, \mathrm{H}-24), 4.57$ (q, 1H, H-16), 7.38-7.41 (m, 5H, Ar-H); ${ }^{13} \mathrm{C}$ NMR (100 MHz, $\left.\mathrm{CDCl}_{3}\right): \delta 158.6\left(\mathrm{C}-2^{\prime}\right), 138.9$ (Ar-C), 129.6 (Ar-C), 128.3 (Ar-C), 128.2 (Ar-C), 110.3 (C-2), 87.2 (C20), 84.5 (C-24), 73.3 (C-16), 70.9 (C-25), 55.6 (C-17), 48.7 (C-5), 48.6 (C-15), 46.5 (C-8), 46.2 (C-4, C-14), 45.6 (C-13), 37.3 (C-22), 34.9 (C-1), 33.1 (C-12), 29.8 (C-19), 27.2 (C-27, C-29), 26.5 (C-26), 
26.1 (C-11, C-18), 25.8 (C-10), 25.4 (C-7), 25.2 (C-30), 23.7 (C-23), 23.6 (C-6), 21.8 (C-21), 20.5 (C28), 19.6 (C-9); HRESIMS $m / z$ calcd for $\mathrm{C}_{38} \mathrm{H}_{53} \mathrm{~N}_{2} \mathrm{O}_{4} 601.4005$, found $601.4015[\mathrm{M}+\mathrm{H}]^{+}$.

(16 $\beta, 20 R, 24 R)-16,24-e p o x y-25-h y d r o x y-2$ '-oxo-4'-phenyl-3'H-pyrimidino[5',6':2,3]cycloartane (10)

White solid; $[\alpha]_{\mathrm{D}}^{25}+34.2^{\circ}(c$ 0.1, MeOH); IR (KBr) vmax $3427(\mathrm{O}-\mathrm{H}), 2939(\mathrm{C}-\mathrm{H}), 1645(\mathrm{C}=\mathrm{O}), 1600(\mathrm{Ar}$ $\mathrm{C}=\mathrm{C}), 1452,1377,1110(\mathrm{C}-\mathrm{O}), 1056 \mathrm{~cm}^{-1} ;{ }^{1} \mathrm{H}$ NMR (400 MHz, $\left.\mathrm{CDCl}_{3}\right): \delta 0.33(\mathrm{~d}, J=4.4 \mathrm{~Hz}, 1 \mathrm{H}, \mathrm{H}-$ 19a), 0.63 (d, $J=4.4 \mathrm{~Hz}, 1 \mathrm{H}, \mathrm{H}-19 \mathrm{~b}), 0.86$ (d, $\left.J=6.4 \mathrm{~Hz}, 3 \mathrm{H}, \mathrm{H}_{3}-21\right), 0.87$ (s, 3H, H3-28), 1.09 (s, $3 \mathrm{H}$, H3-18), 1.06 (s, 6H, H3-26, H3-27), 1.16 (m, 1H, H-7a), 1.37 (s, 3H, H3-30), 1.38 (m, 1H, H-22a), 1.40 (m, 1H, H-7b), 1.48 (m, 1H, H-15a), 1.52 (s, 3H, H3-29), 1.54 (m, 1H, H-23a), 1.76 (m, 2H, H2-6), 1.57 (m, 1H, H-17), 1.60 (m, 2H, H2-12), 1.55 (m, 1H, H-5), 1.85 (m, 1H, H-8), 1.74 (m, 1H, H-22b), 1.88 (d, $J=16.2 \mathrm{~Hz}, 1 \mathrm{H}, \mathrm{H}-1 \mathrm{a}), 1.88$ (m, 1H, H-15b), 1.90 (m, 1H, H-23b), 2.02 (m, 2H, H2-11), 2.03 (m, 1H, H-20), 2.80 (d, $J=16.2$ Hz, 1H, H-1b), 3.56 (dd, 1H, H-24), 4.57 (m, 1H, H-16), 7.33-7.46 (m, 5H, ArH), 12.90 (brs, $1 \mathrm{H},-\mathrm{NH}) ;{ }^{13} \mathrm{C}$ NMR (100 MHz, CDCl 3 ): $\delta 164.4$ (C-3), 158.9 (C-2'), 129.5 (Ar-C), 128.3 (Ar-C), 128.1 (Ar-C), 110.4 (C-2), 82.5 (C-24), 74.8 (C-16), 73.2 (C-25), 57.4 (C-17), 48.5 (C-5), 45.7 (C-14), 45.6 (C-8), 45.5 (C-13), 45.5 (C-4), 45.1 (C-15), 35.4 (C-22), 34.9 (C-1), 32.6 (C-12), 29.8 (C19), 28.8 (C-20), 26.5 (C-29), 25.6 (C-7, C-10), 25.5 (C-27), 25.3 (C-11), 23.8 (C-26), 23.4 (C-23), 23.4 (C-30), 21.8 (C-6), 20.8 (C-21), 19.7 (C-9), 19.3 (C-28), 19.0 (C- 18); HRESIMS m/z calcd for $\mathrm{C}_{38} \mathrm{H}_{53} \mathrm{~N}_{2} \mathrm{O}_{3}$ 585.4056, found $585.4067[\mathrm{M}+\mathrm{H}]^{+}$.

(16 $\beta, 20 R, 24 R)-16,24,25-t r i h y d r o x y-2 '-o x o-4 '-p h e n y l-3 ' H-p y r i m i d i n o[5 ', 6 ': 2,3] c y c l o a r t a n e(12)$ White solid; $[\alpha]_{\mathrm{D}}^{25}+111.0^{\circ}\left(c\right.$ 0.1, MeOH); IR (KBr) $v_{\max } 3406(\mathrm{O}-\mathrm{H}), 2937(\mathrm{C}-\mathrm{H}), 1645(\mathrm{C}=\mathrm{O}), 1598(\mathrm{Ar}$ $\mathrm{C}=\mathrm{C}), 1444,1380 \mathrm{~cm}^{-1} ;{ }^{1} \mathrm{H}$ NMR $\left(400 \mathrm{MHz}, \mathrm{CD}_{3} \mathrm{OD}\right): \delta 0.40(\mathrm{~d}, J=4.2 \mathrm{~Hz}, 1 \mathrm{H}, \mathrm{H}-19 \mathrm{a}), 0.70(\mathrm{~d}, J=$ $4.2 \mathrm{~Hz}, 1 \mathrm{H}, \mathrm{H}-19 \mathrm{~b}), 0.91$ (d, $\left.J=6.4 \mathrm{~Hz}, 3 \mathrm{H}, \mathrm{H}_{3}-21\right), 0.95$ (s, 3H, H3-28), 1.14 (s, 3H, H3-26), 1.16 (s, 3H, H3-18), 1.16 (s, 3H, H3-27), 1.18 (m, 1H, H-22a), 1.20 (m, 1H, H-23a), 1.29 (s, 3H, H3-30), 1.38 (m, 1H, H-15a), 1.42 (m, 1H, H-23b), 1.44 (s, 3H, H3-29), 1.59 (m, 2H, H2-6), 1.60 (m, 1H, H-5), 1.62 (m, 
2H, H2-12), 1.67 (m, 1H, H-17), 1.77 (m, 1H, H-22b), 1.80 (m, 2H, H2-7), 1.86 (m, 1H, H-20), 1.90 (d, J $=16.5 \mathrm{~Hz}, 1 \mathrm{H}, \mathrm{H}-1 \mathrm{a}), 1.97(\mathrm{~m}, 1 \mathrm{H}, \mathrm{H}-8), 2.02\left(\mathrm{~m}, 2 \mathrm{H}, \mathrm{H}_{2}-11\right), 2.04(\mathrm{~m}, 1 \mathrm{H}, \mathrm{H}-15 \mathrm{~b}), 2.85$ (d, $J=16.5$ Hz, 1H, H-1b), 3.36 (dd, 1H, H-24), 4.43 (m, 1H, H-16), 7.49 (m, 5H, Ar-H); ${ }^{13} \mathrm{C}$ NMR (100 MHz, $\left.\mathrm{CD}_{3} \mathrm{OD}\right): \delta 164.0$ (C-3), 159.8 (C-2'), 131.3 (Ar-C), 129.8 (Ar-C), 129.5 (Ar-C), 112.6 (C-2), 78.6 (C24), 73.9 (C-25), 73.4 (C-16), 58.2 (C-17), 50.5 (C-5), 49.3 (C-15), 47.8 (C-14), 46.9 (C-8), 46.5 (C-4), 46.5 (C-13), 35.4 (C-1), 33.9 (C-12), 33.8 (C-22), 30.6 (C-19), 29.9 (C-20), 28.5 (C-6), 27.0 (C-29), 26.9 (C-11), 26.8 (C-23), 25.5 (C-27), 25.5 (C-10), 25.4 (C-26), 24.8 (C-30), 23.7 (C-7), 20.8 (C-28), 20.7 (C-9), 19.9 (C-18), 18.5 (C-21); HRESIMS m/z calcd for $\mathrm{C}_{38} \mathrm{H}_{55} \mathrm{~N}_{2} \mathrm{O}_{4}, 603.4162$, found 603.4169 $[\mathrm{M}+\mathrm{H}]^{+}$

\section{Synthesis of argentatin analogues 13,14 , and 15}

To each of the stirred solutions of $\mathbf{1}(10.0 \mathrm{mg}, 1.0 \mathrm{eq}), \mathbf{2}(10.0 \mathrm{mg}, 1.0 \mathrm{eq}$,$) , and \mathbf{3}(1.0 \mathrm{eq}, 15.0 \mathrm{mg}) \mathrm{in}$ $\mathrm{CH}_{3} \mathrm{CO}_{2} \mathrm{H}(1.0 \mathrm{~mL})$ was added a bromine solution $(1.5 \mathrm{eq}, 2 \mathrm{~mL})$ and the resulting mixtures were stirred at $0^{\circ} \mathrm{C}-25{ }^{\circ} \mathrm{C}$ for $1 \mathrm{~h}$ (TLC control). The reaction mixtures were quenched with (10\%) aq. $\mathrm{NaHCO}_{3}$ solution and extracted with EtOAc $(3 \times 5 \mathrm{~mL})$. The combined EtOAc extracts were dried (anhydrous $\mathrm{Na}_{2} \mathrm{SO}_{4}$ ) and concentrated under reduced pressure. The resulting residues were dissolved in absolute EtOH $(0.5 \mathrm{~mL})$ to which thiourea (1.5 eq) was added and refluxed for $12 \mathrm{~h}$ (TLC control). The reaction mixtures were extracted with EtOAc $(3 \times 5 \mathrm{~mL})$, dried (anhydrous $\left.\mathrm{Na}_{2} \mathrm{SO}_{4}\right)$, and evaporated under reduced pressure. The residues thus obtained were purified by column chromatography using silica gel. Elution with hexane-EtOAc (60-40) followed by $\mathrm{CH}_{2} \mathrm{Cl}_{2}-i$ - $\mathrm{PrOH}(90-10)$ afforded the argentatin analogues 13 (6.5 mg, 58\%), 14 (6.0 mg, 53\%), and $15(5.2 \mathrm{mg}, 46 \%)$.

(16 $\beta, 20 S, 24 R)$-20,24-epoxy-16,25-dihydroxy-2'-amino-1,3-thiazolo-[3,2]cycloartane (13) White solid; $[\alpha]_{\mathrm{D}}^{25}+112\left(c\right.$ 0.1, MeOH); IR (KBr) $v_{\max } 3371(\mathrm{O}-\mathrm{H}), 3209(\mathrm{~N}-\mathrm{H}), 2939(\mathrm{C}-\mathrm{H}), 1624,1531,1377$, 1184, 1053, $732 \mathrm{~cm}^{-1} ;{ }^{1} \mathrm{H}$ NMR (400 MHz, $\left.\mathrm{CDCl}_{3}\right): \delta 0.48(\mathrm{~d}, J=4.6 \mathrm{~Hz}, 1 \mathrm{H}, \mathrm{H}-19 \mathrm{a}), 0.74(\mathrm{~d}, J=4.6$ Hz, 1H, H-19b), 0.88 (m, 1H, H-6a), 0.90 (s, 3H, H3-28), 1.06 (m, 1H, H-7a), 1.10 (s, 3H, H3-30), 1.12 
(s, 3H, H3-26), 1.23 (s, 3H, H3-27), 1.24 (s, 3H, H3-29), 1.27 (s, 3H, H3-18), 1.37 (m, 1H, H-7b), 1.44 (s, 3H, H3-21), 1.51 (m, 1H, H-15a), 1.62 (m, 1H, H-5), 1.67 (m, 1H, H-22a), 1.72 (m, 1H, H-6b), 1.78 (m, 1H, H-8), 1.82 (m, 2H, H2-12), 1.93 (m, 2H, H2-23), 2.01 (d, J=15.8 Hz, 1H, H-1a), 2.04 (m, 1H, H15b), 2.12 (m, 2H, H2-11), 2.14 (d, J=7.5 Hz, 1H, H-17), 2.24 (m, 1H, H-22b), 2.81 (d, J=15.8 Hz, 1H, H-1b), 3.84 (t, $J=7.6 \mathrm{~Hz}, 1 \mathrm{H}, \mathrm{H}-24), 4.60$ (ddd, $J=12.7,7.7,4.9 \mathrm{~Hz}, 1 \mathrm{H}, \mathrm{H}-16), 4.78$ (s, 2H, NH2$\left.2^{\prime}\right) ;{ }^{13} \mathrm{C}$ NMR (100 MHz, $\left.\mathrm{CDCl}_{3}\right): \delta 164.6\left(\mathrm{C}-2^{\prime}\right), 154.6$ (C-3), $117.0(\mathrm{C}-2), 87.2(\mathrm{C}-20), 84.5$ (C-24), 73.4 (C-16), 70.9 (C-25), 55.6 (C-17), 48.8 (C-15), 48.5 (C-5), 46.3 (C-8), 46.6 (C-14), 46.1 (C-13), 39.3 (C-4), 37.3 (C-22), 33.2 (C-12), 32.0 (C-1), 30.4 (C-19), 27.3 (C-27, C-29), 26.2 (C-26), 26.1 (C-7, C-11), 25.7 (C-18), 24.9 (C-10), 23.8 (C-23), 23.0 (C-30), 21.5 (C-21), 21.4 (C-6), 20.6 (C-28), 19.3 (C9); HRESIMS $m / z$ calcd for $\mathrm{C}_{31} \mathrm{H}_{49} \mathrm{~N}_{2} \mathrm{O}_{3} \mathrm{~S} 529.3464$, found $529.3465[\mathrm{M}+\mathrm{H}]^{+}$.

$(16 \beta, 20 R, 24 R)$-16,24-epoxy-25-hydroxy-2'-amino-1,3-thiazolo-[3,2]cycloartane (14) White solid; $[\alpha]_{\mathrm{D}}^{25}$ +54 (c 0.1, $\left.\mathrm{CHCl}_{3}\right)$; IR (KBr) $v_{\max } 3375(\mathrm{~N}-\mathrm{H}), 2943(\mathrm{C}-\mathrm{H}), 1620,1531,1461,1373,1110,1060,736$ $\mathrm{cm}^{-1} ;{ }^{1} \mathrm{H} \mathrm{NMR}\left(400 \mathrm{MHz}, \mathrm{CDCl}_{3}\right): \delta 0.48$ (d, $\left.J=4.4 \mathrm{~Hz}, 1 \mathrm{H}, \mathrm{H}-19 \mathrm{a}\right), 0.74$ (d, J=4.4 Hz, 1H, H-19b), 0.90 (s, 3H, H3-28), 0.92 (d, $\left.J=6.6 \mathrm{~Hz}, 3 \mathrm{H}, \mathrm{H}_{3}-21\right), 1.06$ (m, 1H, H-7a), 1.07 (s, 6H, H3-26, H3-27), 1.11 (s, 3H, H3-30), 1.16 (s, 3H, H3-18), 1.24 (s, 3H, H3-29), 1.36 (m, 1H, H-7b), 1.38 (m, 1H, H-22a), 1.50 (m, 1H, H-15a), 1.56 (m, 1H, H-23a), 1.57 (m, 1H, H-5), 1.60 (m, 1H, H-17), 1.65 (m, 2H, H2-12), 1.74 (m, 1H, H-22b), 1.78 (m, 2H, H2-6), 1.90 (m, 1H, H-15b), 1.92 (m, 1H, H-23b), 1.80 (m, 1H, H-8), 2.02 (d, $J=15.4$ Hz, 1H, H-1a), 2.07 (m, 1H, H-20), 2.10 (m, 2H, H2-11), 2.81 (d, J=15.4 Hz, 1H, H1b), 3.57 (dd, $J=12.4,1.2 \mathrm{~Hz}, 1 \mathrm{H}, \mathrm{H}-24), 4.60$ (m, 1H, H-16), 4.72 (s, 2H, NH2-2'); ${ }^{13} \mathrm{C}$ NMR (100 $\left.\mathrm{MHz}, \mathrm{CDCl}_{3}\right): \delta 164.4\left(\mathrm{C}-2^{\prime}\right), 154.6(\mathrm{C}-3), 117.1(\mathrm{C}-2), 82.5(\mathrm{C}-24), 74.9(\mathrm{C}-16), 73.2(\mathrm{C}-25), 57.4(\mathrm{C}-$ 17), 48.3 (C-5), 46.0 (C-8), 45.8 (C-14), 45.6 (C-13), 45.1 (C-15), 39.3 (C-4), 35.4 (C-22), 32.8 (C-12), 32.0 (C-1), 30.1 (C-19), 28.9 (C-20), 27.3 (C-29), 25.7 (C-11), 25.6 (C-7, C-27), 24.8 (C-10), 23.5 (C23), 23.4 (C-26), 22.9 (C-30), 21.6 (C-6), 20.9 (C-21), 19.8 (C-28), 19.4 (C-9), 19.1 (C- 18); HRESIMS $m / z$ calcd for $\mathrm{C}_{31} \mathrm{H}_{49} \mathrm{~N}_{2} \mathrm{O}_{2} \mathrm{~S}, 513.3515$, found $513.3514[\mathrm{M}+\mathrm{H}]^{+}$. 
$(16 \beta, 20 R, 24 R)-16,24,25$-trihydroxy-2'-amino-1,3-thiazolo-[3,2] cycloartane (15) White solid; $[\alpha]_{\mathrm{D}}^{25}+137$ (c 0.1, MeOH); IR (KBr) v $v_{\max } 3421(\mathrm{O}-\mathrm{H}), 3340(\mathrm{~N}-\mathrm{H}), 2931(\mathrm{C}-\mathrm{H})$, 1627, 1531, 1461, 1380, 1185, $1083 \mathrm{~cm}^{-1} ;{ }^{1} \mathrm{H}$ NMR (400 MHz, $\mathrm{CDCl}_{3}+\mathrm{CD}_{3} \mathrm{OD}$ ): $\delta 0.52$ (d, $\left.J=4.3 \mathrm{~Hz}, 1 \mathrm{H}, \mathrm{H}-19 \mathrm{a}\right), 0.74$ (d, $J=4.3$ Hz, 1H, H-19b), 0.93 (m, 1H, H-6a), 0.96 (s, 3H, H3-28), 0.97 (d, J=6.0 Hz, 3H, H3-21), 1.11 (m, 1H, H-7a), 1.12 (s, 3H, H3-26), 1.17 (m, 1H, H-22a), 1.18 (s, 3H, H3-30), 1.19 (s, 3H, H3-27), 1.22 (s, 3H, H3-18), 1.23 (s, 3H, H3-29), 1.40 (m, 1H, H-7b), 1.42 (m, 1H, H-15a), 1.60 (m, 2H, H2-23), 1.62 (m, 1H, H-5), 1.68 (m, 1H, H-17), 1.72 (m, 2H, H2-12), 1.77 (m, 1H, H-6b), 1.80 (m, 1H, H-8), 1.82 (m, 1H, H22b), 1.91 (m, 1H, H-20), 2.05 (d, J = 16.0 Hz, 1H, H-1a), 2.08 (m, 1H, H-15b), 2.13 (m, 2H, H2-11), $2.80(\mathrm{~d}, J=16.0 \mathrm{~Hz}, 1 \mathrm{H}, \mathrm{H}-1 \mathrm{~b}), 3.38$ (dd, $J=10.4,2.2 \mathrm{~Hz}, 1 \mathrm{H}, \mathrm{H}-24), 4.46$ (m, 1H, H-16), 7.82 (s, 1H, $\left.\mathrm{NH}-1^{\prime}\right) ;{ }^{13} \mathrm{C} \mathrm{NMR}\left(100 \mathrm{MHz}, \mathrm{CDCl}_{3}+\mathrm{CD}_{3} \mathrm{OD}\right): \delta 168.9$ (C-2'), 154.4 (C-3), 116.6 (C-2), $78.6(\mathrm{C}-24)$, 73.9 (C-16), 73.4 (C-25), 58.2 (C-17), 50.6 (C-5), 48.8 (C-15), 47.8 (C-14), 47.7 (C-13), 46.6 (C-8), 40.3 (C-4), 33.8 (C-12), 32.9 (C-1), 31.2 (C-22), 30.0 (C-19), 28.5 (C-20), 27.7 (C-29), 26.1 (C-27), 27.0 (C-23), 25.6 (C-11), 25.4 (C-7, C-10), 23.4 (C-30), 22.8 (C-26), 20.9 (C-6), 20.8 (C-9), 20.7 (C28), 19.9 (C- 18), 18.5 (C-21); HRESIMS $m / z$ calcd for $\mathrm{C}_{31} \mathrm{H}_{51} \mathrm{~N}_{2} \mathrm{O}_{3} \mathrm{~S} 513.3620$, found 531.3607 $[\mathrm{M}+\mathrm{H}]^{+}$.

\section{Synthesis of argentatin analogues 16,17 and 18}

Phenylhydrazine hydrochloride (1.5 eq) was added to stirred solutions of 1 (10.0 mg, $1.0 \mathrm{eq}), 2$ (15.0 $\mathrm{mg}, 1.0 \mathrm{eq})$ and $\mathbf{3}(10.0 \mathrm{mg}, 1.0 \mathrm{eq})$ in $\mathrm{CH}_{3} \mathrm{CO}_{2} \mathrm{H}(1.0 \mathrm{~mL})$ at $25^{\circ} \mathrm{C}$, warmed up to $60{ }^{\circ} \mathrm{C}$ and stirred at this temperature for $5 \mathrm{~h}$ (TLC control). The reaction mixtures were then quenched with $10 \%$ aq. $\mathrm{NaHCO}_{3}$ solution and extracted with EtOAc $(3 \times 5 \mathrm{~mL})$. The combined organic layers were dried (anhydrous $\mathrm{Na}_{2} \mathrm{SO}_{4}$ ) and concentrated under reduced pressure to give crude products, which were purified by column chromatography using silica gel and eluting with toluene-EtOAc (90:10) and $\mathrm{CH}_{2} \mathrm{Cl}_{2}-i-\mathrm{PrOH}$ (96:4) to afford the argentatin analogues 16 (8.6 mg, 74\%), 17 (10.0 mg, 57\%), and 18 (7.8 mg, 68\%). 
(16 $\beta, 20 S, 24 R)$-20,24-epoxy-16,25-dihydroxy-1'H-indolo [3,2-b]cycloartane (16) White solid; $[\alpha]_{\mathrm{D}}^{25}+134$ (c 0.1, MeOH); IR (KBr) $v_{\max } 3417(\mathrm{O}-\mathrm{H}), 2958(\mathrm{C}-\mathrm{H}), 1461,1384,1180,1103,740 \mathrm{~cm}^{-1} ;{ }^{1} \mathrm{H}$ NMR (400 MHz, $\left.\mathrm{CDCl}_{3}\right): \delta 0.50(\mathrm{~d}, J=4.4 \mathrm{~Hz}, 1 \mathrm{H}, \mathrm{H}-19 \mathrm{a}), 0.72(\mathrm{~d}, J=4.4 \mathrm{~Hz}, 1 \mathrm{H}, \mathrm{H}-19 \mathrm{~b}), 0.91(\mathrm{~m}, 1 \mathrm{H}, \mathrm{H}-$ 6a), 0.96 (s, 3H, H3-28), 1.10 (m, 1H, H-7a), 1.13 (s, 3H, H3-26), 1.23 (s, 3H, H3-30), 1.24 (s, 3H, H327), 1.30 (s, 3H, H3-18), 1.32 (s, 3H, H3-29), 1.43 (m, 1H, H-7b), 1.47 (s, 3H, H3-21), 1.57 (m, 1H, H15a), 1.74 (m, 1H, H-22a), 1.77 (m, 1H, H-6b), 1.78 (m, 1H, H-5), 1.84 (m, 2H, H2-12), 1.96 (m, 2H, H2-23), 1.98 (m, 1H, H-8), 2.08 (m, 1H, H-15b), 2.18 (d, $J=7.5 \mathrm{~Hz}, 1 \mathrm{H}, \mathrm{H}-17), 2.26$ (d, $J=15.2 \mathrm{~Hz}$, 1H, H-1a), 2.28 (m, 1H, H-22b), 2.31 (m, 2H, H2-11), 2.90 (d, $J=15.2 \mathrm{~Hz}, 1 \mathrm{H}, \mathrm{H}-1 \mathrm{~b}), 3.86$ (t, $J=7.7$ Hz, 1H, H-24), 4.62 (ddd, $J=12.7,7.7,5.0 \mathrm{~Hz}, 1 \mathrm{H}, \mathrm{H}-16), 7.04$ (t, $J=7.0 \mathrm{~Hz}, 1 \mathrm{H}, \mathrm{H}-4$ '), 7.10 (t, $J=$ $\left.7.0 \mathrm{~Hz}, 1 \mathrm{H}, \mathrm{H}-3^{\prime}\right), 7.29$ (d, $\left.J=7.6 \mathrm{~Hz}, 1 \mathrm{H}, \mathrm{H}-2^{\prime}\right), 7.38$ (d, $\left.J=7.6 \mathrm{~Hz}, 1 \mathrm{H}, \mathrm{H}-5^{\prime}\right), 7.79$ (s, 1H, NH-1'); ${ }^{13} \mathrm{C}$ NMR (100 MHz, CDCl 3 ): $\delta 142.9$ (C-3), 135.8 (C-6'), $127.6\left(\mathrm{C}-7^{\prime}\right), 121.0\left(\mathrm{C}-3^{\prime}\right), 118.9\left(\mathrm{C}-4^{\prime}\right), 117.8$ (C-5'), 110.5 (C-2'), 108.1 (C-2), 87.3 (C-20), 84.5 (C-24), 73.5 (C-16), 70.9 (C-25), 55.6 (C-17), 48.8 (C-15), 48.7 (C-5), 46.7 (C-8), 46.6 (C-14), 46.3 (C-13), 36.5 (C-4), 37.4 (C-22), 33.3 (C-12), 30.6 (C19), 29.9 (C-1), 27.5 (C-29), 27.3 (C-27), 26.2 (C-26), 26.1 (C-7, C-11), 25.7 (C-18), 25.3 (C-10), 24.1 (C-30), 23.8 (C-23), 21.2 (C-6), 21.3 (C-21), 20.6 (C-28), 19.3 (C-9); HRESIMS m/z calcd for $\mathrm{C}_{36} \mathrm{H}_{52} \mathrm{NO}_{3} 546.3947$, found $546.3940[\mathrm{M}+\mathrm{H}]^{+}$.

(16 $\beta, 20 R, 24 R)$-16,24-epoxy-25-hydroxy-1'H-indolo[3,2-b]cycloartane (17) White solid; $[\alpha]_{\mathrm{D}}^{25}+28(c 0.1$, $\mathrm{CHCl}_{3}$ ); IR (KBr) $v_{\max } 3317$ (N-H), $2954(\mathrm{C}-\mathrm{H}), 1465,1384,1157,1056,964,732 \mathrm{~cm}^{-1}$; ${ }^{1} \mathrm{H}$ NMR (400 $\left.\mathrm{MHz}, \mathrm{CDCl}_{3}\right): \delta 0.50$ (d, $\left.J=4.2 \mathrm{~Hz}, 1 \mathrm{H}, \mathrm{H}-19 \mathrm{a}\right), 0.70$ (d, $\left.J=4.2 \mathrm{~Hz}, 1 \mathrm{H}, \mathrm{H}-19 \mathrm{~b}\right), 0.94$ (s, 3H, H3-28), 0.95 (d, $\left.J=6.2 \mathrm{~Hz}, 3 \mathrm{H}, \mathrm{H}_{3}-21\right), 1.08$ (s, 6H, H3-26, H3-27), 1.19 (s, 3H, H3-18), 1.23 (s, 3H, H3-30), 1.24 (m, 1H, H-7a), 1.32 (s, 3H, H3-29), 1.41 (m, 1H, H-22a), 1.43 (m, 1H, H-7b), 1.52 (m, 1H, H-15a), 1.59 (m, 1H, H-23a), 1.61 (m, 1H, H-5), 1.65 (m, 1H, H-17), 1.73 (m, 2H, H2-12), 1.76 (m, 1H, H-22b), 1.78 (m, 2H, H2-6), 1.91 (m, 1H, H-15b), 1.93 (m, 1H, H-23b), 1.97 (m, 1H, H-8), 2.08 (m, 1H, H-20), 2.25 (d, $J=15.4 \mathrm{~Hz}, 1 \mathrm{H}, \mathrm{H}-1 \mathrm{a}), 2.26$ (m, 2H, $\left.\mathrm{H}_{2}-11\right), 2.90$ (d, $\left.J=15.4 \mathrm{~Hz}, 1 \mathrm{H}, \mathrm{H}-1 \mathrm{~b}\right), 3.58$ (dd, $J=$ 
12.6, 2.0 Hz, 1H, H-24), 4.61 (m, 1H, H-16), 7.04 (t, J=7.1 Hz, 1H, H-4'), 7.09 (t, J= 7.0 Hz, 1H, H3'), $7.29\left(\mathrm{~d}, J=7.8 \mathrm{~Hz}, 1 \mathrm{H}, \mathrm{H}-2^{\prime}\right), 7.38$ (d, $\left.J=7.4 \mathrm{~Hz}, 1 \mathrm{H}, \mathrm{H}-5^{\prime}\right), 7.76$ (s, 1H, NH-1'); ${ }^{13} \mathrm{C}$ NMR (100 $\left.\mathrm{MHz}, \mathrm{CDCl}_{3}\right): \delta 142.9(\mathrm{C}-3), 135.8\left(\mathrm{C}^{-6}\right), 127.6\left(\mathrm{C}-7^{\prime}\right), 120.9\left(\mathrm{C}-3^{\prime}\right), 118.9\left(\mathrm{C}^{\prime} 4^{\prime}\right), 117.8\left(\mathrm{C}^{\prime} 5^{\prime}\right), 110.5$ (C-2'), 108.2 (C-2), 82.5 (C-24), 74.9 (C-16), 73.2 (C-25), 57.4 (C-17), 48.5 (C-5), 46.5 (C-8), 45.8 (C14), 45.7 (C-13), 45.2 (C-15), 36.4 (C-4), 35.4 (C-22), 32.8 (C-12), 30.4 (C-19), 29.9 (C-1), 28.9 (C-20), 27.5 (C-29), 25.8 (C-11, C-27), 25.7 (C-7), 24.6 (C-10), 24.0 (C-30), 23.8 (C-26), 23.5 (C-23), 21.3 (C6), 20.9 (C-21), 19.8 (C-28), 19.5 (C-9), 19.1 (C- 18); HRESIMS $m / z$ calcd for $\mathrm{C}_{36} \mathrm{H}_{52} \mathrm{NO}_{2} 530.3998$, found $530.3978[\mathrm{M}+\mathrm{H}]^{+}$.

(16 $\beta, 20 R, 24 R)-16,24,25$-trihydroxy-1'H-indolo[3,2-b] cycloartane (18) White solid; yield 68\% (7.8 mg); $[\alpha]_{\mathrm{D}}^{25}+111.0^{\circ}\left(c\right.$ 0.1, MeOH); IR (KBr) $v_{\max } 3417(\mathrm{O}-\mathrm{H}), 2939(\mathrm{C}-\mathrm{H}), 1465,1380,1180,1053,740 \mathrm{~cm}^{-1}$ ${ }^{1} \mathrm{H}$ NMR (400 MHz, $\left.\mathrm{CDCl}_{3}\right): \delta 0.50(\mathrm{~d}, J=4.2 \mathrm{~Hz}, 1 \mathrm{H}, \mathrm{H}-19 \mathrm{a}), 0.71(\mathrm{~d}, J=4.2 \mathrm{~Hz}, 1 \mathrm{H}, \mathrm{H}-19 \mathrm{~b}), 0.95$ $\left(\mathrm{d}, J=6.1 \mathrm{~Hz}, 3 \mathrm{H}, \mathrm{H}_{3}-21\right), 0.96\left(\mathrm{~s}, 3 \mathrm{H}, \mathrm{H}_{3}-28\right), 0.98$ (m, 1H, H-6a), 1.13 (m, 1H, H-22a), 1.16 (s, 3H, Н3-26), 1.20 (s, 3H, H3-18), 1.21 (s, 3H, H3-27), 1.23 (s, 3H, H3-30), 1.26 (m, 1H, H-7a), 1.32 (s, 3H, Н3-29), 1.38 (m, 1H, H-15a), 1.44 (m, 1H, H-7b), 1.60 (m, 2H, H2-23), 1.64 (m, 1H, H-5), 1.70 (m, 1H, H-17), 1.74 (m, 2H, H2-12), 1.80 (m, 1H, H-6b), 1.90 (m, 1H, H-22b), 1.95 (m, 1H, H-20), 1.98 (m, $1 \mathrm{H}$, H-8), 2.14 (m, 1H, H-15b), 2.26 (d, J=15.5 Hz, 1H, H-1a), 2.28 (m, 2H, H2-11), 2.90 (d, J=15.5 Hz, 1H, H-1b), 3.58 (dd, $J=11.7,2.3$ Hz, 1H, H-24), 4.50 (m, 1H, H-16), 7.03 (t, $\left.J=6.8 \mathrm{~Hz}, 1 \mathrm{H}, \mathrm{H}-4^{\prime}\right)$, 7.10 (t, $\left.J=7.2 \mathrm{~Hz}, 1 \mathrm{H}, \mathrm{H}-3^{\prime}\right), 7.29$ (d, J=7.2 Hz, 1H, H-2'), 7.38 (d, J=7.2 Hz, 1H, H-5'), 7.76 (s, 1H, NH-1'); ${ }^{13} \mathrm{C}$ NMR (100 MHz, $\left.\mathrm{CDCl}_{3}\right): \delta 142.8$ (C-3), 135.8 (C-6'), $127.6\left(\mathrm{C}-7^{\prime}\right), 120.9(\mathrm{C}-3$ '), 118.9 (C-4'), 117.8 (C-5'), 110.5 (C-2'), 108.1 (C-2), 75.2 (C-24), 73.0 (C-16, C-25), 56.8 (C-17), 48.9 (C-5), 48.1 (C-15), 46.7 (C-14), 46.6 (C-8), 45.3 (C-13), 36.6 (C-4), 32.8 (C-12), 31.1 (C-22), 30.4 (C-19), 29.9 (C-1), 27.5 (C-29), 26.8 (C-27), 26.6 (C-20), 26.2 (C-23), 25.9 (C-7), 25.8 (C-11), 24.7 (C-10), 24.1 (C-30), 23.2 (C-26), 21.3 (C-6), 20.2 (C-28), 19.6 (C- 18), 19.5 (C-9), 17.7 (C-21); HRESIMS m/z calcd for $\mathrm{C}_{36} \mathrm{H}_{54} \mathrm{NO}_{3} 548.4104$, found $548.4103[\mathrm{M}+\mathrm{H}]^{+}$. 


\section{Cytotoxicity evaluation}

Cytotoxic activity of test compounds was determined in a panel of human non-small cell lung (NCIH460), human CNS glioma (SF-268), human breast (MCF-7) and normal cells (WI 38) using the dyereduction (MTT) assay as described previously [32]. Doxorubicin and DMSO were used as positive and negative controls, respectively. The test compounds were dissolved in DMSO and diluted to the desired concentrations in culture medium and were added to $96-w e l l$ plates. After $72 \mathrm{~h}$, incubation at $37^{\circ} \mathrm{C}$ in a $\mathrm{CO}_{2}$ incubator, dye solution (MTT [3-(4, 5-dimethylthiazol-2-yl)- 2,5- diphenyl tetrazolium bromide]) was added to each well (1:10 dilution). After agitation, incubation was continued for $4 \mathrm{~h}$ at $37^{\circ} \mathrm{C}$ in a $\mathrm{CO}_{2}$ incubator. The supernatant liquid from each well was carefully removed, and the resulting formazan crystals were dissolved in DMSO. The absorbance in each well was read using a microplate fluorometer (Ex/Em: 570/650).

Acknowledgments This project was supported by the USDA-NIFA (Grant \# 2017-68005-26867; Dr. Kimberly Ogden, PI) and Hatch projects ARZT-1005072 (AALG) and ARZT-1361640-H12-224 (IM), and the University of Arizona College of Agriculture. Any opinions, findings, conclusions, or recommendations expressed in this publication/work are those of the authors and do not necessarily reflect the view of the U.S. Department of Agriculture. We thank Dr. Lijiang Xuan, Shanghai Institute of Materia Medica, Chinese Academy of Sciences, P. R. China for HRMS data.

\section{Compliance with ethical standards}

Conflict of interest I. M. has disclosed financial interests in TEVA Pharmaceuticals Hungary and the University of Debrecen, Hungary, which are unrelated to the subject of the research presented here. All other authors declare that they have no known competing financial interests or personal relationships that could have appeared to influence the work reported in this paper.

Publisher's note Springer Nature remains neutral with regard to jurisdictional claims in published maps and institutional affiliations. 
Supplementary information The online version contains supplementary material available at https://doi.org/10.1007/s00044-Xxx-Xxxxx-x.

\section{References}

1. Butler MS. The role of natural product chemistry in drug discovery. J Nat Prod. 2004;67:2141-2153. https://doi.org/10.1021/np040106y.

2. Newman DJ, Cragg GM. Natural products as sources of new drugs from 1981 to 2014. J Nat Prod. 2016;79:629-661. https://doi.org/10.1021/acs.jnatprod.5b01055.

3. Kingston DGI. Modern natural products drug discovery and its relevance to biodiversity conservation. J Nat Prod. 2011;74:496-511. https://doi.org/10.1021/np100550t.

4. Butler MS. Natural products to drugs: natural product derived compounds in clinical trials. Nat Prod Rep. 2005;22:475-516. https://doi.org/10.1039/B514294F.

5. Newman DJ. Natural products as leads to potential drugs: An old process or the new hope for drug discovery. J Med Chem. 2008;51:2589-2599. https://doi.org/10.1021/jm0704090.

6. Salvador JAR, Moreira VM, Gonçalves BMF, Leal AS, Jing Y. Ursane-type pentacyclic triterpenoids as useful platforms to discover anticancer drugs. Nat Prod Rep. 2012;29:14631479. https://doi.org/10.1039/C2NP20060K.

7. Petronelli A, Pannitteri G, Testa U. Triterpenoids as new promising anticancer drugs. Anticancer Drugs. 2009;20:880-892. https://doi: 10.1097/CAD.0b013e328330fd90.

8. Rahim A, Saito Y, Miyake K, Goto M, Chen CH, Alam G, Natschke SM, Lee KH, Goto KN. Kleinhospitine E and cycloartane triterpenoids from Kleinhovia hospita. J Nat Prod. 2018;81:1619-1627. https://doi.org/10.1021/acs.jnatprod.8b00211.

9. Banjar MFS, Mohamed GA, Shehata IA, Abdallah HM, Shatid AA, Alfaifid MY, Elbehairi SEI, Koshak AE, Ibrahim SRM. Cycloschimperols A and B, new cytotoxic cycloartane 
triterpenoids from Euphorbia schimperi. Phytochem Lett. 2019;32:90-95.

https://doi.org/10.1016/j.phytol.2019.05.008.

10. Vivar AR, Vázquez MM, Matsubara C, Sánchez GP, Nathan PJ. Triterpenes in Parthenium argentatum, structures of argentatins C and D. Phytochemistry. 1990;29:915-918. https://doi: 10.1016/0031-9422(90)80045-I.

11. Komoroski RA, Gregg EC, Shockcor JP, Geckle JM. Identification of guayule triterpenes by two dimensional and multipulse NMR techniques. Magn Reson Chem. 1986;24:534-543. https://doi.org/10.1002/mrc.126024061.

12. Schloman WW, Hively RA, Krishen A, Andrews AM. Guayule byproduct evaluation: extract characterization. J Agric Food Chem. 1983;31:873-876. https://doi:10.1021/jf00118a050.

13. Piana F, Ciulu M, Piné RQ, Sanna G, Carretero, AS, Spano N, Mariani Alberto. Simple and rapid procedures for the extraction of bioactive compounds from guayule leaves. Ind Crops Prod. 2018;116:162-169. https://doi.org/10.1016/j.indcrop.2018.02.057.

14. Maatooq GT, Gamal, AAHE, Furbacher TR, Cornuelle TL, Hoffmann JJ. Triterpenoids from Parthenium argentatum $\times$ P. tomentosa. Phytochemistry. 2002;60:755-760. https://doi.org/10.1016/S0031-9422(02)00166-8.

15. Martinez VM, Martinez R, Perez, GE, Diaz M, Sanchez H. Antimicrobial properties of argentatin A, isolated from Parthenium argentatum. Fitoterapia. 1994;65:371-372.

16. Flores EA, Franco AEB, López PG, Zavaleta LR, Marure RL, Vázquez MM. Argentatin B inhibits proliferation of prostate and colon cancer cells by inducing cell senescence. Molecules. 2015;20:21125-21137. https://doi.org/10.3390/molecules201219757.

17. Calzada L, Salazar EL, Tellez J, Martinez M, Martinez R. Effect of tetracyclic triterpenes (argentatins A, B and D) on the estradiol receptor of hormone-dependent tumors of human breast. Med Sci Res. 1995;23(12):815-816. 
18. Delgado HP, Apan TR, Vázquez MM. Synthesis of argentatin A derivatives as growth inhibitors of human cancer cell lines in vitro. Bioorg Med Chem Lett. 2005;15:1005-1008. https://doi.org/10.1016/j.bmcl.2004.12.038.

19. Delgado HP, Compadre CM, Apan TR, Fambuena MJM, Compadre RL, Wegman PO, Vázquez MM. Synthesis and comparative molecular field analysis (CoMFA) of argentatin B derivatives as growth inhibitors of human cancer cell lines. Bioorg Med Chem. 2006;14:1889-1901. https://doi.org/10.1016/j.bmc.2005.10.038.

20. Singh R, Choudin A. An overview of biological importance of pyrimidines. World J Pharm Pharm Sci. 2014;3(12):574-597.

21. Mallavadhan UV, Chandrashekhar M, Nayak VL, Ramakrishna S. Synthesis and anticancer activity of novel fused pyrimidine hybrids of myrrhanone $\mathrm{C}$, a bicyclic triterpene of Commiphora mukul gum resin. Mol divers. 2015;19:745-757.

https://doi.org/10.1007/s11030-015-9621-3.

22. Chandrashekhar M, Sagarika Gudem, Ramakrishna S, Uppuluri VM. Synthesis and antiinflammatory activity of some novel pyrimidine hybrids of myrrhanone A, a bicyclic triterpene of Commiphora mukul gum resin. Monatsh Chem. 2017;148:2183-2193. https://doi.org/10.1007/s00706-017-2024-7.

23. Nevagi RJ. Biological and medicinal significance of 2-aminothiazoles. Der Pharm Lett. 2014;6(5):134-150. https://www.scholarsresearchlibrary.com/articles/biological-andmedicinal-significance-of-2aminothiazoles.pdf.

24. Borková L, Frydrych I, Jakubcová N, Adámek R, Lišková B, Gurská S, Medvedíková M, Hajdúch M, Urban M. Synthesis and biological evaluation of triterpenoid thiazoles derived from betulonic acid, dihydrobetulonic acid, and ursonic acid. Eur J Med Chem. 2020;185:111806. https://doi.org/10.1016/j.ejmech.2019.111806. 
25. Sayed MTE, Hamdy NA, Osman DA, Ahmed KM. Indoles as anticancer agents. Adv Mod Oncol Res. 2015;1:20-35. http://dx.doi.org/10.18282/amor.v1.11.12.

26. Bosquesi PS, Melo TRF, Vizioli EO, dos Santos JL, Chung MC. Anti-inflammatory drug design using a molecular hybridization approach. Pharmaceuticals. 2011;4:1450-1474. https://doi.org/10.3390/ph4111450.

27. Ren Y, Ma Y, Cherukupalli S, Tavis JE, Menéndes-Arias L, Liu X, Zhan P. Discovery and optimization of benzenesulfonamides-based hepatitis B virus capsid modulators via contemporary medicinal chemistry strategies. Euro J Med Chem. 2020;206:112714. https://doi.org/10.1016/j.ejmech.2020.112714.

28. Xu YM, Madasu C, Liu MX, Wijeratne EMK, Dierig D, White B, Molnàr I, Gunatilaka AAL. Cycloartane and lanostane-type triterpenoids from the resin of Parthenium argentatum AZ-2, a byproduct of guayule rubber production. ACS Omega. 2021;6:15484-15498. https://doi.org/10.1021/acsomega.1c01714.

29. Barlin GB, Brown DJ, Fenn MD. Carbon-13 nuclear magnetic resonance spectra in the identification of N-, O- or S-methyl derivatives of some tautomeric hydroxy and mercapto nitrogen heterocycles. Aust J Chem. 1984;37:2391-2395.

https://doi.org/10.1071/CH9842391

30. Baji Á, Kiss T, Wölfling J, Kovács D, Igaz N, Gopisetty MK, Kiricsi M, Franka É. Multicomponent access to androstano-arylpyrimidines under microwave conditions and evaluation of their anti-cancer activity in vitro. J Steroid Biochem. Mol Biol. 2017;172:7988. https://doi.org/10.1016/j.jsbmb.2017.06.001.

31. Wu Q, Wang R, Shi Y, Li W, Li M, Chen P, Pan B, Wang Q, Li C, Wang J, Sun G, Sun X, $\mathrm{Fu} \mathrm{H}$. Synthesis and biological evaluation of panaxatriol derivatives against myocardial 
ischemia/reperfusion injury in the rat. Eur J Med Chem. 2020;185:111729.

https://doi.org/10.1016/j.ejmech.2019.111729.

32. Wijeratne EMK, Bashyal BP, Liu MX, Rocha DD, Gunaherath, GMKB, U'Ren JM, Gunatilaka MK, Arnold AE, Whitesell L, Gunatilaka AAL. Geopyxins A-E, ent-kaurane diterpenoids from endolichenic fungal strains Geopyxis aff. majalis and Geopyxis sp. AZ0066: Structure-activity relationships of geopyxins and their analogues. J Nat Prod. 2012;75:361-369. https://doi.org/10.1021/np200769q. 
Table 1

Cytotoxicity $\left(\mathrm{IC}_{50}\right)$ data of argentatins $\mathrm{A}-\mathrm{C}(\mathbf{1}-\mathbf{3})$ and their semi-synthetic analogues (7-12) against selected cancer cell lines and human normal cells ${ }^{\mathrm{a}}$

\begin{tabular}{ccccc}
\hline Compound & \multicolumn{3}{c}{ Cell lines $^{\mathrm{b}}$} \\
\cline { 2 - 5 } & NCI-H460 & SF-268 & MCF-7 & WI-38 \\
\hline $\mathbf{1}$ & $31.2 \pm 0.0$ & $35.0 \pm 0.2$ & $32.8 \pm 1.2$ & $41.4 \pm 2.8$ \\
$\mathbf{2}$ & $17.5 \pm 0.9$ & $31.2 \pm 1.7$ & $23.1 \pm 0.4$ & $21.0 \pm 2.9$ \\
$\mathbf{3}$ & $>35.0$ & $>35.0$ & $>35.0$ & $>35.0$ \\
$\mathbf{7}$ & $4.0 \pm 0.8$ & $>10.0$ & $3.8 \pm 1.0$ & $9.8 \pm 1.0$ \\
$\mathbf{8}$ & $4.4 \pm 0.7$ & $>10.0$ & $4.0 \pm 0.4$ & $>10.0$ \\
$\mathbf{9}$ & $3.8 \pm 0.8$ & $>10.0$ & $5.7 \pm 0.6$ & $5.1 \pm 1.1$ \\
$\mathbf{1 0}$ & $3.9 \pm 0.3$ & $7.6 \pm 0.7$ & $3.6 \pm 0.1$ & $>10.0$ \\
$\mathbf{1 2}$ & $3.4 \pm 0.7$ & $>10$ & $4.0 \pm 0.8$ & $0.8 \pm 0.1$ \\
Doxorubicin & $0.1 \pm 0.0$ & $0.4 \pm 0.1$ & $0.3 \pm 0.1$ & \\
\hline
\end{tabular}

${ }^{a}$ Results are expressed as Inhibition Concentration $\left(\mathrm{IC}_{50}\right)$ value in $\mu \mathrm{M}$; Doxorubicin and DMSO were used as positive and negative controls, respectively; Analogues 11 and 13-17 were inactive against all 3 cancer cell lines and normal cells up to $10.0 \mu \mathrm{M}$ concentration. ${ }^{\text {b}}$ Key: NCI-H460 = human non-small cell lung cancer; SF-268 = human CNS cancer (glioma); MCF-7 = human breast cancer; WI-38 = normal human primary fibroblast cells. 

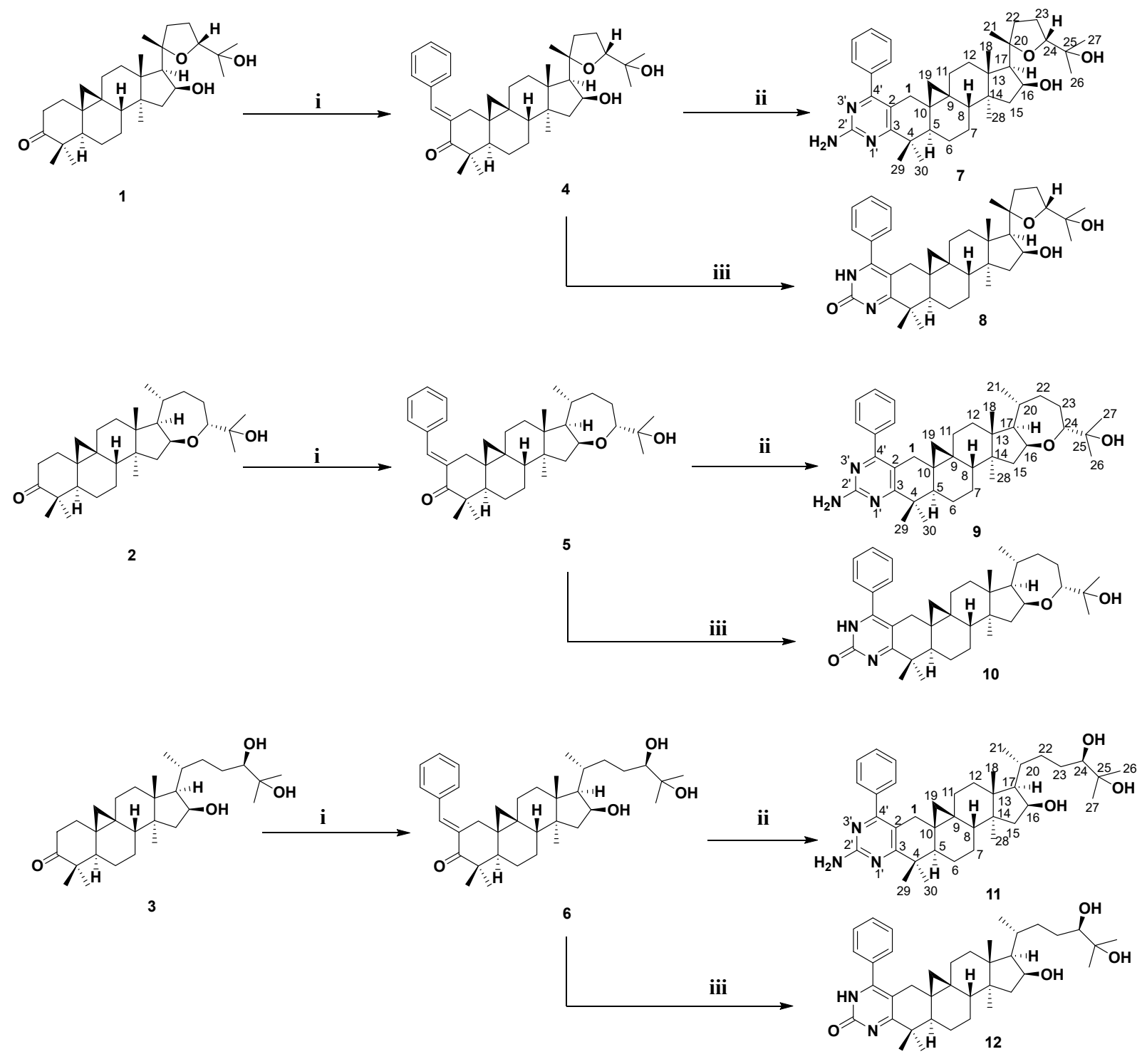

Scheme 1. Synthesis of pyrimidine analogues of argentatins A-C (1-3). Reagents and conditions: i) EtOH, $\mathrm{KOH}$, benzaldehyde, $45^{\circ} \mathrm{C}, 12 \mathrm{~h}$; ii) a. $\mathrm{EtOH}, \mathrm{KOH}, \mathrm{NH}_{2} \mathrm{C}(=\mathrm{NH}) \mathrm{NH}_{2} . \mathrm{HCl}$, reflux, $12 \mathrm{~h}$; b. 1,4 dioxane, DDQ, $25^{\circ} \mathrm{C}, 1$ h; iii) a. EtOH, $\mathrm{KOH}, \mathrm{NH}_{2} \mathrm{C}(=\mathrm{O}) \mathrm{NH}_{2}$ reflux, $12 \mathrm{~h}$; b. 1,4 dioxane, DDQ, $25^{\circ} \mathrm{C}, 1 \mathrm{~h}$. 

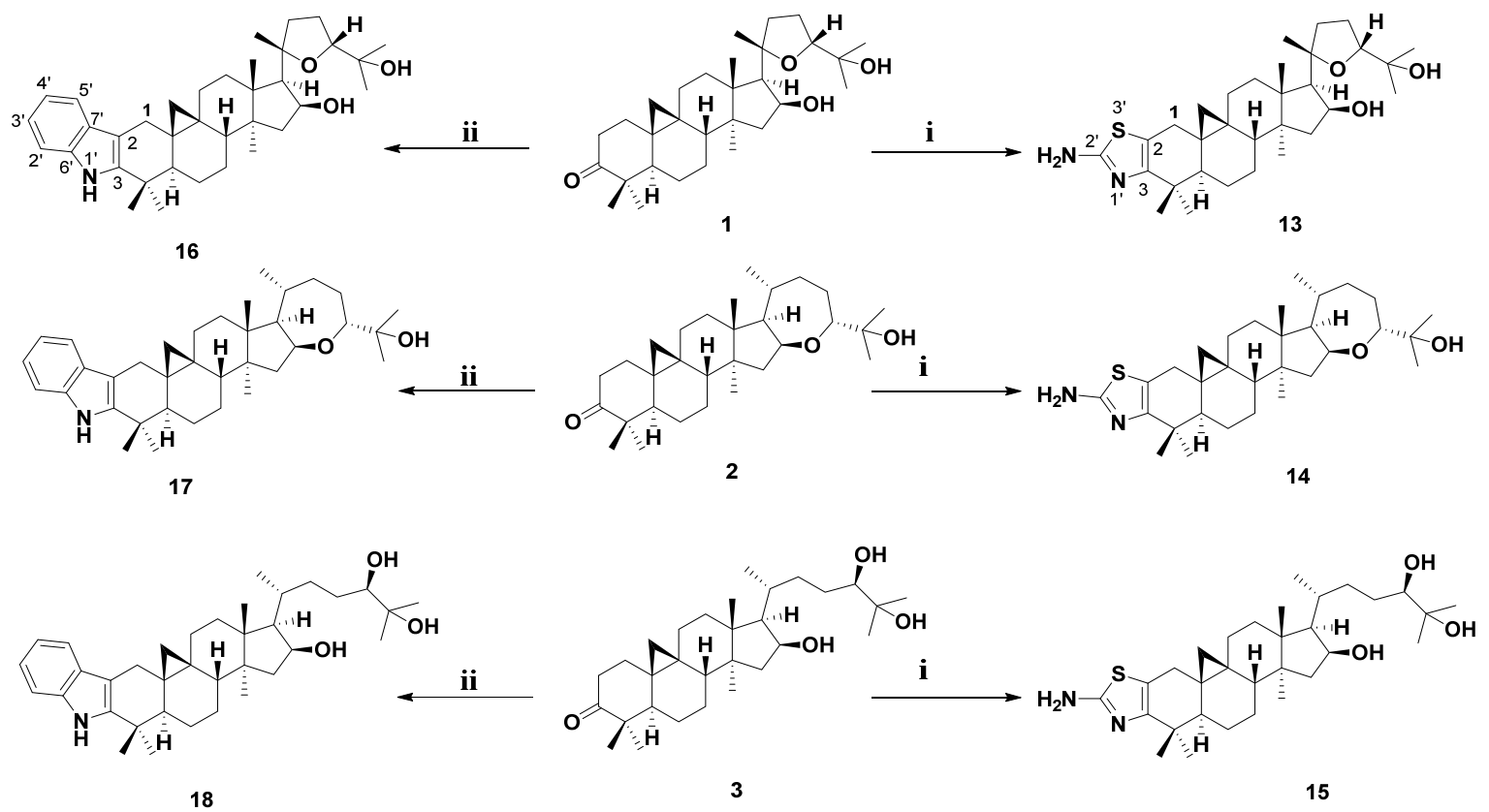

Scheme 2. Synthetic routes to thiazole and indole analogues of argentatins A-C. Reagents and conditions: $\mathrm{i}$. a. $\mathrm{CH}_{3} \mathrm{COOH}(1.0 \mathrm{~mL}), \mathrm{Br}_{2}(1.5 \mathrm{eq}), 0{ }^{\circ} \mathrm{C}-25^{\circ} \mathrm{C}, 1 \mathrm{~h}$; b. Ethanol $(0.5 \mathrm{~mL}), \mathrm{NH}_{2} \mathrm{C}(=\mathrm{S}) \mathrm{NH}_{2}$ (1.5 eq), reflux, $12 \mathrm{~h}$; ii. $\mathrm{CH}_{3} \mathrm{COOH}(1.0 \mathrm{~mL}), \mathrm{PhNHNH}_{2} \cdot \mathrm{HCl}(1.5 \mathrm{eq}), 50^{\circ} \mathrm{C}, 5 \mathrm{~h}$. 


\section{Supplementary Files}

This is a list of supplementary files associated with this preprint. Click to download.

- SupplementaryData.pdf 